
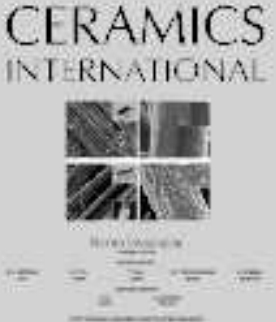


\section{$Q_{\text {SEARCH }} \equiv{ }_{\text {MENU }}$}

Home (https://www.elsevier.com/) > Journals (https://www.elsevier.com/catalog?producttype=journals)

(https://www.elsevier.c Ceramics International (https://www.journals.elsevier.com:443/cerami...

om) > Editorial Board (https://www.journals.elsevier.com:443/ceramics-international/editorial-board)

Submit Your Paper

Supports Open Access (https://www.elsevier.com/journals/ceramics-international/0272-8842/open-access-options)

View Articles (https://www.sciencedirect.com/science/journal/o2728842)

Guide for Authors

Abstracting/ Indexing (http://www.elsevier.com/journals/ceramics-international/o272-8842/abstracting-indexing)

Track Your Paper

Order Journal (https://www.elsevier.com/journals/institutional/ceramics-international/0272-8842)

Journal Metrics

> CiteScore: 6.1 (i)

Impact Factor: 3.830 (i)

5-Year Impact Factor: 3.513 (i)

Source Normalized Impact per Paper (SNIP): 1.310 (i)

SCImago Journal Rank (SJR): 0.891 (i)

$>$ View More on Journal Insights

\section{Your Research Data}

> Share your research data (https://www.elsevier.com/authors/author-resources/research-data)

\section{Related Links}

$>$ Author Stats (1)

$>$ Researcher Academy

$>$ Author Resources (https://www.elsevier.com/authors/author-resources)

$>$ Try out personalized alert features 


\section{Ceramics International - Editorial Board}

\section{General Editor}

\section{P. Vincenzini}

World Academy of Ceramics, National Research Council, Faenza, Italy

\section{Editors-in-Chief}

\section{R.K. Bordia}

Clemson University, Clemson, South Carolina, United States

\section{Z.Y. Fu}

Ministry of Education of the Peoples Republic of China Changjiang Scholar Program, Wuhan, China

\section{T. Ohji}

National Institute of Advanced Industrial Science and Technology Advanced Manufacturing Research Institute, Tsukuba, Japan

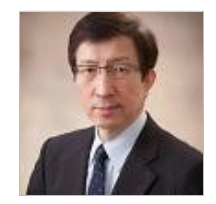

V.C. Pandolfelli (https://www.journals.elsevier.com:443/ceramics-international/editorial-board/vc-pandolfelli) Federal University of Sao Carlos Department of Materials Engineering, SAO CARLOS, Brazil

\section{R. Riedel}

Technical University of Darmstadt Department of Materials and Earth Sciences, Darmstadt, Germany

\section{Associate Editor}

Z.J. Yu

Xiamen University, Xiamen, China 


\section{Editorial Board}

\section{Q SEARCH $\equiv$}

\section{H. Adair}

Pennsylvania State University, University Park, Pennsylvania, PA 15228, United States (https://www.elsevier.c

om)

D. Agrawal (https://www.journals.elsevier.com:443/ceramics-international/editorial-board/dagrawal)

Pennsylvania State University, University Park, PA, United States

\section{A. Akbar}

The Ohio State University Department of Materials Science and Engineering, 295 Watts Hall, 2041 College Road, Columbus, Ohio, 43210-1124, United States

\section{R. Asthana}

University of Wisconsin at Stout Department of Engineering and Technology, 326 Fryklund Hall, Menomonie, Wisconsin, , United States

\section{M.W. Barsoum}

Drexel University Department of Materials Science and Engineering, 3141 Chestnut Street, Philadelphia, Pennsylvania, 19104, United States

\section{J.P. Bennett}

US Department of Energy, Washington, OR, United States

\section{G. Bertrand}

Graduate National School of Chemical and Technological Engineering, 31077, Toulouse, France

\section{K. Byrappa}

University of Mysore Department of Studies in Earth Science, P.B. 21, 570 006, Mysore, India

\section{T. Chartier}

Institut de Recherche sur les Ceramiques, 12, rue Atlantis, 87068, Limoges, France

Professor Paolo Colombo, Dr. ing. (https://www.journals.elsevier.com:443/ceramics-international /editorial-board/professor-paolo-colombo-dr-ing)

University of Padova Department Industrial Engineering, Via Marzolo 9, 35131, Padova, Italy

\section{R. Danzer}

University of Mining, Leoben, Austria

\section{B. Derby}

The University of Manchester, M13 9PL, Manchester, United Kingdom

\section{A. Dominguez-Rodriguez}

University of Seville Department of Condensed Matter Physics, Apartado 1065, 41080, Sevilla, Spain 
National Research Council Institute of Photonics and Nanotechnologies, Trento, Italy (https://www.elsevier.c

om)

\section{M.F. Ferreira}

University of Aveiro, Campus Universitário, 3810-193, Aveiro, Portugal

\section{J.R. Frade}

University of Aveiro Department of Economics Management Industrial Engineering and Tourism, 3810-191, Aveiro, Portugal

\section{N. Frage}

Ben-Gurion University of the Negev, 84105, Be'er Sheva, Israel

\section{W.L. Gladfelter}

University of Minnesota Department of Chemistry, 207 Pleasant Street S.E, Minneapolis, Minnesota, 55455-0431, United States

\section{T. Graule}

Empa Materials Science and Technology, CH-860o, Dübendorf, Switzerland

\section{H.J. Hannink}

CSIRO Australian Manufacturing and Materials Precinct, Normandy Road, Clayton, 3168, Australia

\section{T. Ishikawa}

Sanyo-Onoda City University, 756-o884, Sanyoonoda, Japan

\section{S.J.L. Kang}

Korea Advanced Institute of Science and Technology, South Korea

\section{Kawashita}

Tohoku University International Research Institute of Disaster Science, Sendai, Japan

\section{D.K. Kim}

Korea Advanced Institute of Science and Technology, 335 Gwahangno (373-1 Guseong-dong), Yuseong-gu, 305-701, Daejeon, Korea, Republic of

\section{H-D. Kim}

Korea Institute of Materials Science Engineering Ceramics Research Department, 531 Changwondaero, 641-831, Changwon, Korea, Republic of

\section{Y.-W. Kim}

University of Seoul, Dongdaemun-gu, 02504, Seoul, Korea, Republic of

\section{J. Knowles}

University College London Eastman Dental Institute, 256 Grays Inn Road, WC1X 8LD, London, United Kingdom

\section{W. Krenkel}


Wuhan University of Technology, 430070, Wuhan, China

(https://www.elsevier.c

om)

\section{H.T. Lin}

Guangdong University of Technology - University Town Campus, 510006, Guangzhou, China

J. Lis

AGH University of Science and Technology Faculty of Materials Science and Ceramics, Al. Mickiewicza Adama 30, 30-059, Krakow, Poland

\section{L.M. Llanes Pitarch}

Polytechnic University of Catalonia Department of Materials Science and Metallurgy, ETSEIB, avinguda Diagonal 647, 08028, Barcelona, Spain

\section{P. Miele}

University of Montpellier, 34095, Montpellier, France

\section{Naito}

Osaka University Joining and Welding Research Institute, 11-1 Mihogaoka, 567-0047, Ibaraki-shi, Japan

\section{A.P. Nosov}

M N Mikheev Institute of Metal Physics of the Ural Branch of the Russian Academy of Sciences, 18 S. Kovalevskaya St., 620990, Ekaterinburg, Russian Federation

\section{J. Poirier}

Orleans University, Orleans, France

\section{S. Ramesh}

University of Malaya, 50603, Kuala Lumpur, Malaysia

\section{I.E. Reimanis}

Colorado School of Mines, Golden, Colorado, 80401-1887, United States

\section{K. Rezwan}

University of Bremen, 28359, Bremen, Germany

\section{R.E. Riman}

Rutgers The State University of New Jersey, New Brunswick, New Jersey, NJ 08854, United States

\section{A.S. Rogachev}

Russian Academy of Sciences, Russian Federation

\section{F. Rosei}

National Institute for Scientific Research Energy Materials and Telecommunications Research Centre, Varennes, J3 X 1S2, Quebec, Canada

\section{Y. Sakka}


ELSEVIEP Stockholm University Department of Materials and Environmental Chemistry, S-106 91, Stockholm, Sweden

(https://wwetsegieffnund
om)

University of Florida Department of Materials Science and Engineering, 225 Rhines Hall, P.O. Box 116400, Gainesville, Florida, 32611-6400, United States

\section{Singh}

NASA John H Glenn Research Center, MS 106-5 Ceramic Branch, Cleveland, Ohio, OH 44135-3191, United States

\section{G. Srinivasan}

Oakland University, Rochester, Michigan, 48309-4401, United States

\section{Suvorov}

Jozef Stefan Institute, 1000, Ljubljana, Slovenia

\section{T. Troczynski}

The University of British Columbia, Vancouver, V6T 1Z4, British Columbia, Canada

\section{W.H. Tuan}

National Taiwan University Department of Materials and Science Engineering, No. 1, Sec. 4, Roosevelt Road, 10617, Taipei, Taiwan

\section{A. Vinu}

Newcastle University, NE1 7RU, Newcastle, United Kingdom

\section{Wang}

Tongji University College of Design and Innovation, Shanghai, China

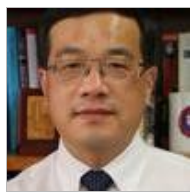

\section{S. Yin}

Tohoku University, 2-1-1 Katahira, Aoba-ku, 980-8577, Sendai, Japan

\section{N. Zhou}

Henan University of Technology, Luoyang, China

\section{Y. Zhou}

Chinese Academy of Sciences, China

\section{Y. Zhou}

Harbin Institute of Technology Institute of Advanced Ceramics, 92 West Dazhi Street, Nan'gang District, 1500o1, Harbin, China

\section{Ceramics International}

\section{Readers}

View Articles 
ELSEVIEAAuthor Information Pack (https://www.elsevier.com/journals/ceramics-international/0272-8842?generatepdf=true)

ELSEVIEP Submit Your Paper Track Your Paper

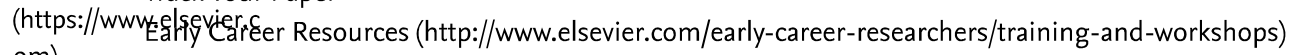

om) Rights and Permissions (https://www.elsevier.com/about/policies/copyright/permissions)

Webshop

Support Center

Librarians (https://www.elsevier.com/librarians)

Ordering Information and Dispatch Dates (http://www.elsevier.com/journals/ceramics-international/o272-8842/order-journal)

Abstracting/ Indexing (http://www.elsevier.com/journals/ceramics-international/o272-8842/abstracting-indexing)

Editors (http://www.elsevier.com/editors/home)

Publishing Ethics Resource Kit (http://www.elsevier.com/editors/perk)

Guest Editors (https://www.elsevier.com/editors/guest-editors)

Support Center

Reviewers (http://www.elsevier.com/reviewers/home)

Reviewer Guidelines (https://www.elsevier.com/reviewers/how-to-review)

Log in as Reviewer

Reviewer Recognition (https://www.elsevier.com/reviewers/becoming-a-reviewer-how-and-why\#recognizing)

Support Center

Advertisers Media Information (https://www.elsevier.com/advertisers)

Societies (http://www.elsevier.com/societies/home)

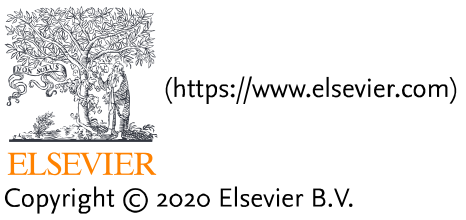

Careers (https://www.elsevier.com/careers/careers-with-us) - Terms and Conditions (https://www.elsevier.com/legal/elsevier-website-terms-and-

conditions) - Privacy Policy (https://www.elsevier.com/legal/privacy-policy)

Cookies are used by this site. To decline or learn more, visit our Cookies page.

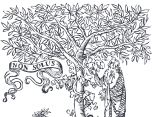

(https://www.elsevier.com) Q RELX Group ${ }^{\text {TM }}$ (http://www.reedelsevier.com/)

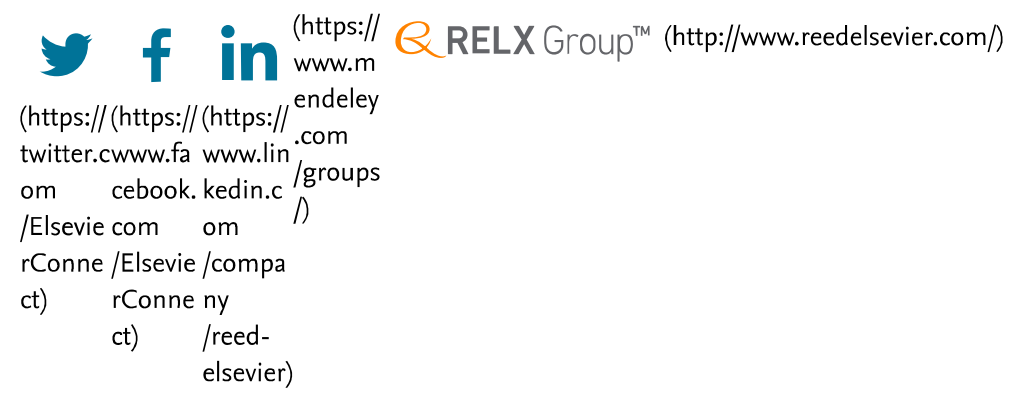




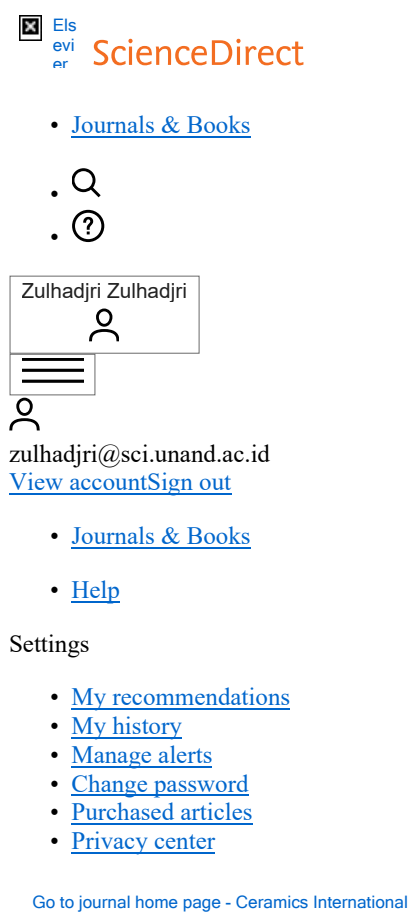

\section{Ceramics International}

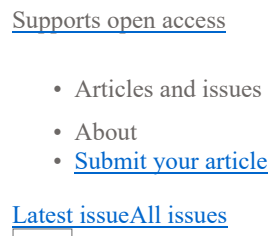

- Articles and issues

- About

- $\underline{\text { Submit your article }}$

Latest issueAll issues

\section{Volume 46, Issue 5}

Pages 5507-7000 (1 April 2020)

〈previous vol/issue

Next vol/issue

Actions for 100 selected articles

Select all $/$ Deselect all

\begin{tabular}{|l|l|}
$\boldsymbol{\Downarrow}_{\text {Download PDFs }}$ & $\boldsymbol{\uparrow}_{\text {Export citations }}$ \\
\hline
\end{tabular}

$\square$ Show all article previewsShow all article previews

\section{Contents}

- $\underline{\text { Reviews }}$

- Original Articles

- Short Communications

- Corrigendum

Receive an update when the latest issues in this journal are published 


\section{Reviews}

1. $\square$ select article Doped $\mathrm{ZnO}$ nanostructures with selected elements - Structural, morphology and optical properties: A review

Review articleAbstract only

Doped ZnO nanostructures with selected elements - Structural, morphology and optical properties: A review

Boshra Ghanbari Shohany, Ali Khorsand Zak

Pages 5507-5520

$\omega_{\text {View PDF }}$

Article preview ${ }^{\vee}$

2. $\square$ select article Review of perovskite-structure related cathode materials for solid oxide fuel cells

Review articleAbstract only

$\underline{\text { Review of perovskite-structure related cathode materials for solid oxide fuel cells }}$

\begin{tabular}{l} 
Paramvir Kaur, K. Singh \\
Pages 5521-5535 \\
$\boldsymbol{\downarrow}$ View PDF \\
\hline Article preview \\
\end{tabular}

3. $\square$ select article Wood-derived ultra-high temperature carbides and their composites: A review

Review articleAbstract only

Wood-derived ultra-high temperature carbides and their composites: A review

Min Yu, Guo-jun Zhang, Theo Saunders

Pages 5536-5547

$\downarrow_{\text {View PDF }}$

Article preview ${ }^{\vee}$

\section{Original Articles}

1. $\square$ select article Qualitative description on channel cracking in ceramic type coatings due to substrate tension using 3D discrete element method

Research articleAbstract only

Qualitative description on channel cracking in ceramic type coatings due to substrate tension using 3D discrete element method

M.A. Ghasemi, S.R. Falahatgar

Pages 5548-5565

${ }_{\text {View PDF }}$

Article preview $\checkmark$

2. $\square$ select article Ternary $\mathrm{ZnO} / \mathrm{ZnAl}<\mathrm{sub}>2</$ sub $>\mathrm{O}<\mathrm{sub}>4</$ sub $>/ \mathrm{Al}<$ sub $>2</$ sub $>\mathrm{O}<$ sub $>3</$ sub $>$ composite nanofiber as photocatalyst for conversion of $\mathrm{CO}<\mathrm{sub}>2</$ sub $>$ and $\mathrm{CH}<\mathrm{sub}>4</$ sub $>$

Research articleAbstract only

$\underline{\text { Ternary } \mathrm{ZnO} / \mathrm{ZnAl}}{ }_{2} \underline{\mathrm{O}}_{4} / \mathrm{Al}_{2} \underline{\mathrm{O}}_{3}$ composite nanofiber as photocatalyst for conversion of $\mathrm{CO}_{2} \underline{\text { and }}_{\mathrm{CH}_{4}}$

Pouran Moradipour, Farzad Dabirian, Mahsa Moradipour

Pages 5566-5574

עView PDF

Article preview $\vee$

3. $\square$ select article The formation mechanism of pores and unbonding in the $\mathrm{Al}<$ sub $>2</$ sub $>\mathrm{O}<$ sub $>3</$ sub $>/ \mathrm{Al}<$ sub $>2</$ sub $>\mathrm{O}<$ sub $>3</$ sub $>$ joints brazed by $50 \mathrm{Bi}<$ sub $>2</$ sub $>0<$ sub $>3</$ sub $>-35 \mathrm{~B}<$ sub $>2</$ sub $>0<$ sub $>3</$ sub $>-15 \mathrm{ZnO}$ glass

Research articleAbstract only

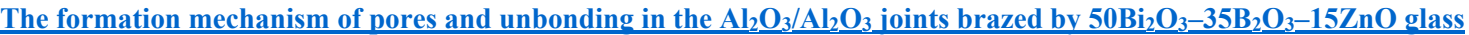

Wenhang Niu, Qianqian Chen, Panpan Lin, Jingyi Cui, ... Xiaoming Duan

Pages 5575-5585

$\Perp_{\text {View PDF }}$

Article preview $\vee$

4. $\square$ select article Preparation and formation mechanism of $\mathrm{C} / \mathrm{C}-\mathrm{SiC}$ composites using polymer-Si slurry reactive melt infiltration 
Research articleAbstract only

Preparation and formation mechanism of $\mathrm{C} / \mathrm{C}-\mathrm{SiC}$ composites using polymer-Si slurry reactive melt infiltration

Wenjian Guo, Yicong Ye, Shuxin Bai, Li'an Zhu, Shun Li

Pages 5586-5593

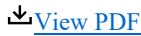

Article preview $\vee$

5. $\square$ select article Processing and thermal characterization of polymer derived $\mathrm{SiCN}(\mathrm{O})$ and $\mathrm{SiOC}$ reticulated foams

Research articleAbstract only

Processing and thermal characterization of polymer derived $\mathrm{SiCN}(\mathrm{O})$ and $\mathrm{SiOC}$ reticulated foams

Balanand Santhosh, Cekdar Vakifahmetoglu, Emanuel Ionescu, Andreas Reitz, ... Gian Domenico Sorarù Pages 5594-5601

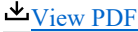

Article preview $\vee$

6. $\square$ select article Characteristics of polycarbosilanes produced under different synthetic conditions and their influence on SiC fibers: Part I

Research articleAbstract only

Characteristics of polycarbosilanes produced under different synthetic conditions and their influence on SiC fibers: Part I

Seokhun Jang, Seong Gun Bae, Dong-Geun Shin, Kwangyoun Cho, ... Yoonjoo Lee

Pages 5602-5609

实View PDF

Article preview $\vee$

7. $\square$ select article Low-temperature exfoliated graphene oxide incorporated with different types of natural rubber latex: Electrical and morphological properties and its capacitance performance

Research articleAbstract only

Low-temperature exfoliated graphene oxide incorporated with different types of natural rubber latex: Electrical and morphological properties and its capacitance performance

M.D. Nurhafizah, A.A. Aziz, A.B. Suriani, A. Mohamed, T. Soga

Pages 5610-5622

齿iew PDF

Article preview ${ }^{\vee}$

8. $\square$ select article Material removal and interactions between an abrasive and a SiC substrate: A molecular dynamics simulation study

Research articleAbstract only

Material removal and interactions between an abrasive and a SiC substrate: A molecular dynamics simulation study

Van-Thuc Nguyen, Te-Hua Fang

Pages 5623-5633

$\stackrel{\Downarrow \text { View PDF }}{ }$

Article preview ${ }^{\vee}$

9. $\square$ select article Silver diffusion and mechanism of $\mathrm{CaO}-\mathrm{MgO}-\mathrm{SiO}<\mathrm{sub}>2</$ sub $>$ glass ceramics in millimeter-wave properties

Research articleAbstract only

$\underline{\text { Silver diffusion and mechanism of } \mathrm{CaO}-\mathrm{MgO}-\mathrm{SiO}_{2}}$ glass ceramics in millimeter-wave properties

Kuei-Chih Feng, Ming-Wei Chu, Chun-An Lu, Yoshiyuki Iizuka, ... Chung-Ya Tsao

Pages 5634-5640

$\downarrow_{\text {View PDF }}$

Article preview ${ }^{\vee}$

10. $\square$ select article Full characterization for material constants of a promising KNN-based lead-free piezoelectric ceramic

Research articleAbstract only

$\underline{\text { Full characterization for material constants of a promising KNN-based lead-free piezoelectric ceramic }}$

Liao Qiao, Guo Li, Hong Tao, Jiagang Wu, ... Fei Li

Pages 5641-5644

$\stackrel{\boldsymbol{w}_{\text {View PDF }}}{ }$ 
Article preview ${ }^{\vee}$

11. $\square$ select article Thermal stability and heat flux investigation of neutron-irradiated nanocrystalline silicon carbide (3C-SiC) using DSC spectroscopy

Research articleAbstract only

Thermal stability and heat flux investigation of neutron-irradiated nanocrystalline silicon carbide (3C-SiC) using DSC spectroscopy

Elchin M. Huseynov

Pages 5645-5648

$\boldsymbol{\Perp}_{\text {View PDF }}$

Article preview ${ }^{\top}$

12. $\square$ select article Experimental investigation on high temperature wettability and structural behaviour of $\mathrm{SAW}$ fluxes using $\mathrm{MgO}-\mathrm{TiO}<\mathrm{sub}>2</$ sub $>$ $-\mathrm{SiO}<\mathrm{sub}>2</$ sub $>$ and $\mathrm{Al}<\mathrm{sub}>2</$ sub $>\mathrm{O}<\mathrm{sub}>3</$ sub $>-\mathrm{MgO}-\mathrm{SiO}<$ sub $>2</$ sub $>$ flux system

Research articleAbstract only

Experimental investigation on high temperature wettability and structural behaviour of SAW fluxes using $\mathrm{MgO}^{-}-\mathrm{TiO}_{2}-\underline{\mathrm{SiO}}_{2} \underline{\text { and }}$ $\underline{\mathrm{Al}}_{2} \underline{\mathrm{O}}_{3}-\mathrm{MgO}-\mathrm{SiO}_{2} \underline{\text { flux system }}$

Lochan Sharma, Jagdish Kumar, Rahul Chhibber

Pages 5649-5657

$\stackrel{\Perp}{\text { View PDF }}$

Article preview ${ }^{\vee}$

13. $\square$ select article Modulations in relaxor nature due to $\mathrm{Sr}<\sup >2+</$ sup $>$ doping in $0.68 \mathrm{PMN}-0.32 \mathrm{PT}$ ceramic

Research articleOpen access

Modulations in relaxor nature due to $\mathrm{Sr}^{2+}$ doping in 0.68PMN-0.32PT ceramic

Pius Augustine, Muralidhar Miryala, Shibnath Samanta, S. Pavan Kumar Naik, ... M.S. Ramachandra Rao Pages 5658-5664

$\boldsymbol{\Psi}_{\text {Download PDF }}$

Article preview $\vee$

14. $\square$ select article Local structure and evolution of phase transformation in

$\mathrm{Bi}<$ sub $>0.4871</$ sub $>\mathrm{Na}<$ sub $>0.4871</$ sub $>$ La $<$ sub $>0.0172</$ sub $>$ TiO $<$ sub $>3</$ sub $>-$ BaTiO $<$ sub $>3</$ sub $>$ materials

Research articleAbstract only

Local structure and evolution of phase transformation in $\mathrm{Bi}_{0.4871} \mathrm{Na}_{0.4871} \mathrm{La}_{0.0172} \mathrm{TiO}_{3}-\mathrm{BaTiO}_{3}$ materials

Atipong Bootchanont, Thanachai Boonchuduang, Atchara Khamkongkaeo, Thanit Saisopa, ... Rattikorn Yimnirun Pages 5665-5670

$\Perp_{\text {View PDF }}$

Article preview ${ }^{\vee}$

15. $\square$ select article Effect of defect states in the optical and magnetic properties of nanocrystalline NiO synthesised in a single step by an aerosol process

Research articleAbstract only

Effect of defect states in the optical and magnetic properties of nanocrystalline NiO synthesised in a single step by an aerosol process

Nandhini J. Usharani, S.S. Bhattacharya

Pages 5671-5680

$\downarrow$ View PDF

Article preview $\checkmark$

16. $\square$ select article High energy storage density and stable fatigue resistance of

$\mathrm{Na}<$ sub $>0.46</$ sub $>$ Bi $<$ sub $>0.46</$ sub $>$ Ba $<$ sub $>0.05</$ sub $>$ La $<$ sub $>0.02</$ sub $>$ Zr $<$ sub $>0.03</$ sub $>$ Ti $<$ sub $>0.97-<$ em $>$ x $</$ em $></$ sub $>$ Sn $<$ sub $><$ em $>$ x $</$ em $></$ s ceramics

Research articleAbstract only

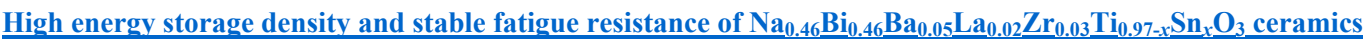

Arun Kumar Yadav, Huiqing Fan, Benben Yan, Chao Wang, ... Shuren Wang

Pages 5681-5688

$\stackrel{\Perp}{\text { View PDF }}$

Article preview ${ }^{\vee}$ 
17. $\square$ select article Preparation, microstructure and electrical property of

$\mathrm{GdSmZr}<$ sub $>2</$ sub $>\mathrm{O}<$ sub $>7</$ sub $>-(\mathrm{Li}<$ sub $>0</$ sub $><$ sub $>$. $</$ sub $><$ sub $>52</$ sub $>$ Na $<$ sub $>0.48</$ sub $>$ ) $<$ sub $>2</$ sub $>$ CO $<$ sub $>3</$ sub $>$ composite electrolyte via carbonate infiltration

Research articleAbstract only

Preparation, microstructure and electrical property of $\mathrm{GdSmZr}_{2} \underline{\mathrm{O}}_{7}-\left(\mathrm{Li}_{0.52} \mathrm{Na}_{0.48}\right)_{2} \mathrm{CO}_{3}$ composite electrolyte via carbonate infiltration

Yu-Jun Jin, Zhan-Guo Liu, Zhao-Ying Ding, Gui Cao, Jia-Hu Ouyang

Pages 5689-5694

$\Perp_{\text {View PDF }}$

Article preview ${ }^{\vee}$

18. $\square$ select article Study on the three-dimensional topography of the machined surface in laser-assisted machining of $\mathrm{Si}<$ sub $>3</$ sub $>\mathrm{N}<$ sub $>4</$ sub $>$ ceramics under different material removal modes

Research articleAbstract only

Study on the three-dimensional topography of the machined surface in laser-assisted machining of $\mathrm{Si}_{3} \mathrm{~N}_{4}$ ceramics under different material removal modes

Yezhuang Pu, Yugang Zhao, Haiyun Zhang, Guoyong Zhao, ... Panpan Song

Pages 5695-5705

$\Perp_{\text {View PDF }}$

Article preview ${ }^{\vee}$

19. $\square$ select article Flower-shaped $\mathrm{ZnO}$ nanomaterials for low-temperature operations in $\mathrm{NO}<\mathrm{sub}>\mathrm{X}</$ sub $>$ gas sensors

Research articleAbstract only

Flower-shaped ZnO nanomaterials for low-temperature operations in NOx gas sensors

Srijita Nundy, Tae-yil Eom, Jun-gu Kang, Jaehyun Suh, ... Hoo-Jeong Lee

Pages 5706-5714

$\boldsymbol{\Perp}_{\text {View PDF }}$

Article preview ${ }^{\vee}$

20. $\square$ select article On the electric conduction of $\mathrm{ZrO}<\mathrm{sub}>2</$ sub $>$ in the steady stage of flash sintering

Research articleAbstract only

On the electric conduction of $\mathrm{ZrO}_{2}$ in the steady stage of flash sintering

Shichen Lu, Jinling Liu, Gang Shao, Wen Liu, ... Linan An

Pages 5715-5718

עView PDF

Article preview ${ }^{\vee}$

21. $\square$ select article Effects of $\mathrm{B}<$ sub $>2</$ sub $>0<$ sub $>3</$ sub $>-\mathrm{Li}<$ sub $>2</$ sub $>$ CO $<$ sub $>3</$ sub $>-$ SiO $<$ sub $>2</$ sub $>-$ ZnO glass on properties of $\mathrm{Li}<\mathrm{sub}>0.43</$ sub $>\mathrm{Zn}<\mathrm{sub}>0.27</$ sub $>\mathrm{Ti}<$ sub $>0.13</$ sub $>\mathrm{Fe}<$ sub $>2.17</$ sub $>0<$ sub $>4</$ sub $>$ ferrites sintered at low temperatures

Research articleAbstract only

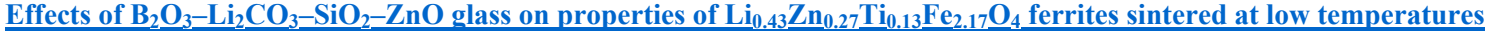

Xiaoyi Wang, Zhiyong Zhong, Zhenwei Chen, Huaiwu Zhang, ... Yulong Liao

Pages 5719-5724

崖iew PDF

Article preview $\vee$

22. $\square$ select article Enhanced photocatalytic hydrogen evolution over $\mathrm{TiO}<$ sub $>2</$ sub $>/$ g- $\mathrm{C}<$ sub $>3</$ sub $>\mathrm{N}<$ sub $>4</$ sub $>2 \mathrm{D}$ heterojunction coupled with plasmon Ag nanoparticles

Research articleAbstract only

Enhanced photocatalytic hydrogen evolution over $\mathrm{TiO}_{2} / \mathrm{g}_{-} \mathrm{C}_{3} \underline{\mathrm{N}}_{4} \underline{2 \mathrm{D}}$ heterojunction coupled with plasmon Ag nanoparticles

Yuhao Yang, Chan Lu, Juan Ren, Xiaolong Li, ... Xia Zhao

Pages 5725-5732

$\downarrow_{\text {View PDF }}$

Article preview ${ }^{\vee}$

23. $\square$ select article The superlattice structure and self-adaptive performance of $\mathrm{C}-\mathrm{Ti} / \mathrm{MoS}<\mathrm{sub}>2</$ sub $>$ composite coatings

Research articleAbstract only 
$\underline{\text { The superlattice structure and self-adaptive performance of } \mathrm{C}-\mathrm{Ti} / \mathrm{MoS}} 2 \underline{\mathrm{S}}_{2}$ composite coatings

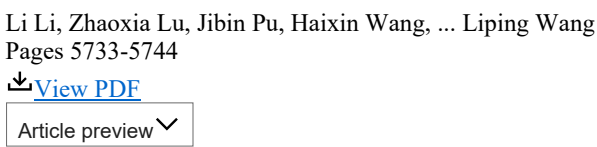

24. $\square$ select article Effect of Mo addition mode on the microstructure and mechanical properties of TiC-high Mn steel cermets

Research articleAbstract only

Effect of Mo addition mode on the microstructure and mechanical properties of TiC-high Mn steel cermets

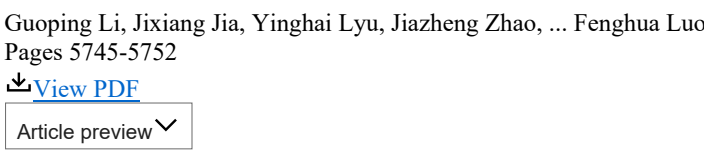

25. $\square$ select article Effects of $\mathrm{TiO}<\mathrm{sub}>2</$ sub $>$ additive on ultra-low-loss $\mathrm{MgO}-\mathrm{LiF}$ microwave dielectric ceramics Research articleAbstract only

Effects of $\mathrm{TiO}_{2}$ additive on ultra-low-loss $\mathrm{MgO}-\mathrm{LiF}$ microwave dielectric ceramics

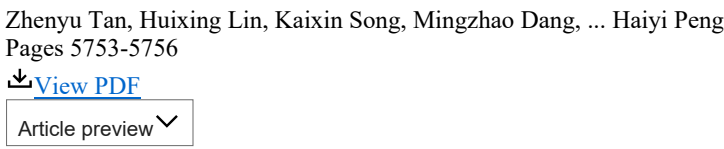

26. $\square$ select article Synthesis of red mud derived M-type barium hexaferrites with tuneable coercivity

Research articleAbstract only

Synthesis of red mud derived M-type barium hexaferrites with tuneable coercivity

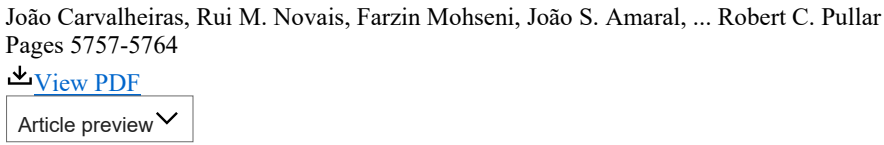

27. $\square$ select article Plasma-enhanced atomic layer deposition of gallium nitride thin films on fluorine-doped tin oxide glass substrate for future photovoltaic application

Research articleAbstract only

Plasma-enhanced atomic layer deposition of gallium nitride thin films on fluorine-doped tin oxide glass substrate for future photovoltaic application

Peng Qiu, Huiyun Wei, Yunlai An, Qixin Wu, ... Xinhe Zheng

Pages 5765-5772

$\Perp_{\text {View PDF }}$

Article preview $\vee$

28. $\square$ select article Combined role of $\mathrm{SiC}$ particles and $\mathrm{SiC}$ whiskers on the characteristics of spark plasma sintered $\mathrm{ZrB}<\mathrm{sub}>2</ \mathrm{sub}>$ ceramics Research articleAbstract only

Combined role of $\mathrm{SiC}$ particles and $\mathrm{SiC}$ whiskers on the characteristics of spark plasma sintered $\mathrm{Zr} \mathrm{B}_{2}$ ceramics

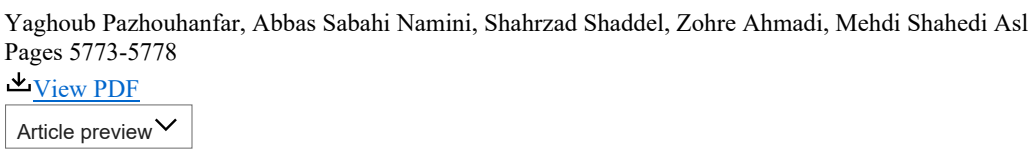

29. $\square$ select article Effect of $\mathrm{Al}<\mathrm{sub}>2</$ sub $>\mathrm{O}<\mathrm{sub}>3</$ sub $>$ particles on mechanical and tribological properties of Al-Mg dual-matrix nanocomposites Research articleAbstract only

$\underline{\text { Effect of } \mathrm{Al}_{2}} \underline{\mathrm{O}}_{3}$ particles on mechanical and tribological properties of $\mathrm{Al}-\mathrm{Mg}$ dual-matrix nanocomposites

M.A. Eltaher, A.Wagih, A. Melaibari, A. Fathy, G. Lubineau Pages 5779-5787

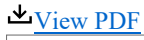

Article preview ${ }^{\vee}$ 
30. $\square$ select article The effect of Ag nanoparticles content on dielectric and microwave absorption properties of $\beta$-SiC

Research articleAbstract only

$\underline{\text { The effect of Ag nanoparticles content on dielectric and microwave absorption properties of } \beta-\mathrm{SiC}}$

Bo Wei, Jintang Zhou, Zhengjun Yao, Azhar Ali Haidry, ... Wenjing Chen

Pages 5788-5798

崖iew PDF

Article preview ${ }^{\vee}$

31. $\square$ select article Influence of carbonation on the low-temperature consolidation by Spark Plasma Sintering of carbonated calcium phosphate bioceramics

Research articleAbstract only

Influence of carbonation on the low-temperature consolidation by Spark Plasma Sintering of carbonated calcium phosphate bioceramics

C. Ortali, I. Julien, C. Drouet, E. Champion

Pages 5799-5810

$\boldsymbol{\Perp}_{\text {View PDF }}$

Article preview ${ }^{\vee}$

32. $\square$ select article Nano-porous carbon materials derived from different biomasses for high performance supercapacitors

Research articleAbstract only

Nano-porous carbon materials derived from different biomasses for high performance supercapacitors

Huafang Yang, Xiaoyan Sun, Han Zhu, Yimin Yu, ... Qitu Zhang

Pages 5811-5820

$\boldsymbol{\Perp}_{\text {View PDF }}$

Article preview $\vee$

33. $\square$ select article $\mathrm{Mn}<\mathrm{sub}><\mathrm{em}>\mathrm{x}</$ em $></$ sub $>\mathrm{Co}<\mathrm{sub}>3<\mathrm{em}>-\mathrm{x}</$ em $></$ sub $>\mathrm{O}<$ sub $>4</$ sub $>$ spinel coatings: Controlled synthesis and high temperature oxidation resistance behavior

Research articleAbstract only

$\mathrm{Mn}_{x} \mathrm{Co}_{3-x} \underline{\mathrm{O}}_{4}$ spinel coatings: Controlled synthesis and high temperature oxidation resistance behavior

Zhengjun Shen, Ju Rong, Xiaohua Yu

Pages 5821-5827

神iew PDF

Article preview ${ }^{\vee}$

34. $\square$ select article Dielectric, thermal and mechanical properties of hybrid PMMA/RGO/Fe $<$ sub $>2</$ sub $>0<$ sub $>3</$ sub $>$ nanocomposites fabricated by in-situ polymerization

Research articleAbstract only

Dielectric, thermal and mechanical properties of hybrid PMMA/RGO/Fe $2 \mathrm{O}_{3}$ nanocomposites fabricated by in-situ polymerization

Yasir Ul-Haq, Imran Murtaza, Sadaf Mazhar, Rizwan Ullah, ... Shahid Amin

Pages 5828-5840

$\boldsymbol{w}_{\text {View PDF }}$

Article preview ${ }^{\vee}$

35. $\square$ select article Study of $(\mathrm{La}, \mathrm{Sr})(\mathrm{Ti}, \mathrm{Ni}) \mathrm{O}<\mathrm{sub}>3-\delta</ \mathrm{sub}>$ materials for symmetrical Solid Oxide Cell electrode - Part B: Conditions of Ni exsolution

Research articleAbstract only

Study of (La,Sr)(Ti,Ni)O ${ }_{3-\delta}$ materials for symmetrical Solid Oxide Cell electrode - Part B: Conditions of Ni exsolution

Charline Arrivé, Thibaud Delahaye, Olivier Joubert, Gilles H. Gauthier

Pages 5841-5849

$\Perp_{\text {View PDF }}$

Article preview $\vee$

36. $\square$ select article Size controlled synthesis of well-distributed nano-silver on hydroxyapatite using alkanolamine compounds

Research articleAbstract only

Size controlled synthesis of well-distributed nano-silver on hydroxyapatite using alkanolamine compounds 
Arniati Labanni, Zulhadjri, Dian Handayani, Yutaka Ohya, Syukri Arief

Pages 5850-5855

$\stackrel{\downarrow}{\text { View PDF }}$

Article preview ${ }^{\vee}$

37. $\square$ select article $\mathrm{AlB}<\mathrm{sub}>12</$ sub $>$-AlB $<$ sub $>12</$ sub $>\mathrm{C}<$ sub $>2</$ sub $>$-TiB $<$ sub $>2</$ sub $>$ hard and tough composites synthesized by reactive plasma activated sintering

Research articleAbstract only

$\underline{\mathrm{AlB}}_{12}-\underline{\mathrm{AlB}}_{12} \underline{\mathrm{C}}_{2}-\underline{T i B}_{2}$ hard and tough composites synthesized by reactive plasma activated sintering

Guoqiang Luo, Peng Fan, Kejia Kang, Rong Tu, ... Lianmeng Zhang

Pages 5856-5862

עView PDF

Article preview ${ }^{\vee}$

38. $\square$ select article Green emitting $\mathrm{Ba}<$ sub $>1.5</$ sub $>$ Lu $<$ sub $>1.5</$ sub $>$ Al $<$ sub $>3.5</$ sub $>$ Si $<$ sub $>1.5</$ sub $>0<$ sub $>12</$ sub $>$ : Ce $<$ sup $>3+</$ sup $>$ phosphor with high thermal emission stability for warm WLEDs and FEDs

Research articleAbstract only

Green emitting Ba Ba $_{1.5} \underline{L u}_{1.5} \mathrm{Al}_{3.5} \mathrm{Si}_{1.5} \mathrm{O}_{12}: \mathrm{Ce}^{3+}$ phosphor with high thermal emission stability for warm WLEDs and FEDs

Xiaoshuang Li, Chengjue Guo, Hongwei Wang, Yeqing Chen, ... Qingguang Zeng

Pages 5863-5870

$\Perp_{\text {View PDF }}$

Article preview ${ }^{\vee}$

39. $\square$ select article Local atomic disorder and temperature dependence of saturation magnetization in yttrium iron garnet

Research articleAbstract only

Local atomic disorder and temperature dependence of saturation magnetization in yttrium iron garnet

R. Peña-Garcia, Y. Guerra, D.M. Oliveira, A. Franco, E. Padrón-Hernández

Pages 5871-5875

עView PDF

Article preview ${ }^{\vee}$

40. $\square$ select article New observations on wear characteristics of solid $\mathrm{Al}<$ sub $>2</$ sub $>\mathrm{O}<$ sub $>3</$ sub $>/$ Si $<$ sub $>3</$ sub $>\mathrm{N}<$ sub $>4</$ sub $>$ ceramic tool in high speed milling of additive manufactured Ti6Al4V

Research articleAbstract only

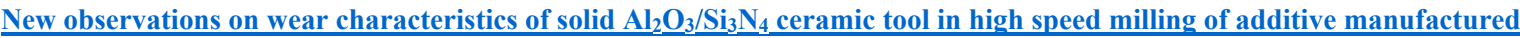
Ti6Al4V

Jiaqiang Dang, Heng Zhang, Weiwei Ming, Qinglong An, Ming Chen

Pages 5876-5886

$\boldsymbol{\Perp}_{\text {View PDF }}$

Article preview $\vee$

41. $\square$ select article Optimizing graphene-TiO $<$ sub $>2</$ sub $>$ interface properties via Fermi level modulation for photocatalytic degradation of volatile organic compounds

Research articleAbstract only

Optimizing graphene- $\mathrm{TiO}_{2}$ interface properties via Fermi level modulation for photocatalytic degradation of volatile organic compounds

Zhen Zhou, Junxian Gao, Guangsheng Zhang, Yuming Dong, ... Jinze Lyu

Pages 5887-5893

$\stackrel{\Perp}{\text { View PDF }}$

Article preview ${ }^{V}$

42. $\square$ select article Electrical and impedance spectroscopy properties of hydrothermally synthesized

$\mathrm{Ba}<$ sub $>0.2</$ sub $>$ Sr $<$ sub $>0.8-\mathrm{y}</$ sub $>$ La $<$ sub $>\mathrm{y}</$ sub $>\mathrm{Fe}<$ sub $>12</$ sub $>\mathrm{O}<$ sub $>19</$ sub $>(\mathrm{y}=0.2-0.8)$ nanorods

Research articleAbstract only

Electrical and impedance spectroscopy properties of hydrothermally synthesized $\mathrm{Ba}_{0.2} \mathrm{Sr}_{0.8-\mathrm{v}} \mathrm{La}_{\mathrm{y}} \mathrm{Fe}_{12} \underline{\mathrm{O}}_{19}(\mathrm{y}=0.2-0.8)$ nanorods

N. Raghuram, T. Subba Rao, K. Chandra Babu Naidu

Pages 5894-5906

$\Perp_{\text {View PDF }}$ 
Article preview ${ }^{\vee}$

43. $\square$ select article Incompatible magnetic and dielectric properties of

$\mathrm{BiCu}<$ sub $>3-\mathrm{x}</$ sub $>\mathrm{Mn}<$ sub $>\mathrm{x}</$ sub $>\mathrm{Ti}<$ sub $>4-\mathrm{y}</$ sub $>\mathrm{M}<$ sub $>\mathrm{y}</$ sub $>\mathrm{O}<$ sub $>12</$ sub $>(\mathrm{x}=0$ \& $0.5 ; \mathrm{y}=1 \& 1.5$ and $\mathrm{M}=\mathrm{Fe} \& \mathrm{Mn})$

Research articleAbstract only

Incompatible magnetic and dielectric properties of $\mathrm{BiCu}_{3-\mathrm{x}} \mathrm{Mn}_{\mathrm{x}} \underline{\mathrm{Ti}}_{4-\mathrm{y}} \mathrm{M}_{\mathrm{y}} \underline{\mathrm{O}}_{12}(\mathrm{x}=0$ \& $0.5 ; \mathrm{y}=1 \& 1.5$ and $\mathrm{M}=\mathrm{Fe} \& \mathrm{Mn})$

Ariful Haque, Ashish Shukla, Uma Dutta, Debamalya Ghosh, ... Md Motin Seikh

Pages 5907-5912

$\stackrel{\Perp}{\text { View PDF }}$

Article preview ${ }^{\vee}$

44. $\square$ select article Synthesis and characterization of $\mathrm{GaNb}<\mathrm{sub}>11</$ sub $>\mathrm{O}<\mathrm{sub}>29</$ sub $>$ @ for high-performance lithium-ion battery

Research articleAbstract only

$\underline{\text { Synthesis and characterization of GaNb}}{ }_{11} \mathrm{O}_{29} @ \mathrm{C}$ for high-performance lithium-ion battery

Zhen Wang, Runtian Zheng, Yuhang Li, Haoxiang Yu, ... Jie Shu

Pages 5913-5919

$\boldsymbol{w}_{\text {View PDF }}$

Article preview ${ }^{\vee}$

45. $\square$ select article Structural, dielectric and magnetic properties of ( $\mathrm{ZnFe}<$ sub $>2</$ sub $>0<$ sub $>4</$ sub $>$ /Polystyrene) nanocomposites synthesized by microemuslion technique

Research articleAbstract only

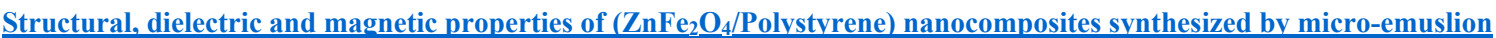
technique

Qaiser Abbas, G. Murtaza, Nawaz Muhammad, Mohsin Ishfaq, ... Muhammad Zahir Iqbal

Pages 5920-5928

$\downarrow_{\text {View PDF }}$

Article preview ${ }^{\vee}$

46. $\square$ select article $\mathrm{Al}<\mathrm{sub}>2</ \mathrm{sub}>\mathrm{O}<\mathrm{sub}>3</ \mathrm{sub}>-\mathrm{CaO}$ macroporous ceramics containing hydrocalumite-like phases

Research articleAbstract only

\section{$\underline{\mathrm{Al}}_{2} \mathrm{O}_{3}-\mathrm{CaO}$ macroporous ceramics containing hydrocalumite-like phases}

O.H. Borges, T. Santos, V.R. Salvini, V.C. Pandolfelli

Pages 5929-5936

$\boldsymbol{w}_{\text {View PDF }}$

Article preview $\vee$

47. $\square$ select article Microstructure and mechanical property of $\mathrm{SiC}$ whisker coating modified $\mathrm{C} / \mathrm{C}$ composite $/ \mathrm{Ti}<\mathrm{Sub}>2</$ sub $>\mathrm{AlNb}$ alloy dissimilar joints prepared by transient liquid phase diffusion bonding

Research articleAbstract only

Microstructure and mechanical property of $\mathrm{SiC}$ whisker coating modified $\mathrm{C} / \mathrm{C}$ composite/Ti $2 \mathrm{AlNb}$ alloy dissimilar joints prepared by transient liquid phase diffusion bonding

Wang Jie, Feng Yang, Zhang Fuqi, Xiong Qinglian, ... Xiong Qinghai

Pages 5937-5945

${ }_{\text {View PDF }}$

Article preview ${ }^{\vee}$

48. $\square$ select article Microstructure and interface-adhesion of thermally sprayed continuous gradient elastic modulus FeCrAl-ceramic coatings

Research articleAbstract only

Microstructure and interface-adhesion of thermally sprayed continuous gradient elastic modulus FeCrAl-ceramic coatings

Qiaolei Li, Chunming Deng, Lu Li, Jianguo Lü, ... Jiansheng Lu

Pages 5946-5959

$\stackrel{\Perp}{\text { View PDF }}$

Article preview ${ }^{\vee}$

49. $\square$ select article Lychee-like $\mathrm{ZnO} / \mathrm{ZnFe}<\mathrm{sub}>2</ \mathrm{sub}>\mathrm{O}<\mathrm{sub}>4</$ sub $>$ core-shell hollow microsphere for improving acetone gas sensing performance Research articleAbstract only 
Lychee-like $\mathrm{ZnO} / \mathrm{ZnFe}_{2} \underline{O}_{4}$ core-shell hollow microsphere for improving acetone gas sensing performance

Yu Hu, Hong Wang, Di Liu, Guo Lin, ... Xiaohua Liu

Pages 5960-5967

$\boldsymbol{w}_{\text {View PDF }}$

Article preview ${ }^{\vee}$

50. $\square$ select article High pressure modifications in amorphous boron suboxide: An ab initio study

Research articleAbstract only

High pressure modifications in amorphous boron suboxide: An ab initio study

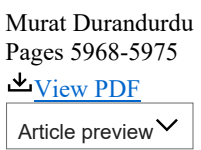

51. $\square$ select article Preparation of oxygen vacancy-controllable $\mathrm{CeO}<$ sub $>2</$ sub $>$ by electrotransformation of a $\mathrm{CeCl}<$ sub $>3</$ sub $>$ solution and its oxidation mechanism

Research articleAbstract only

Preparation of oxygen vacancy-controllable $\mathrm{CeO}_{2}$ by electrotransformation of a $\mathrm{CeCl}_{3}$ solution and its oxidation mechanism

Jiang Liu, Zhihe Dou, Ting-an Zhang

Pages 5976-5982

${ }_{\text {View PDF }}$

Article preview ${ }^{\vee}$

52. $\square$ select article Microstructure evolution in high density AZO ceramic sputtering target fabricated via multistep sintering

Research articleAbstract only

Microstructure evolution in high density AZO ceramic sputtering target fabricated via multistep sintering

Payam Rajabi Kalvani, Samaneh Shapouri, Ali Reza Jahangiri, Yousef Seyed Jalili

Pages 5983-5992

$\stackrel{\downarrow}{\text { View PDF }}$

Article preview ${ }^{\vee}$

53. $\square$ select article Influence of different coating structures on the oxidation resistance of $\mathrm{MoSi}<\mathrm{sub}>2</$ sub $>$ coatings

Research articleAbstract only

Influence of different coating structures on the oxidation resistance of $\mathrm{MoSi}_{2}$ coatings

L. Liu, H.Q. Zhang, H. Lei, H.Q. Li, ... C. Sun

Pages 5993-5997

$\Downarrow_{\text {View PDF }}$

Article preview ${ }^{\vee}$

54. $\square$ select article Studying crystal-field splitting difference of Eu<sup $>3+</$ sup $>$ ions from orthorhombic to cubic $\mathrm{Ca}<$ sub $>4</$ sub $>\mathrm{Al}<$ sub $>6</$ sub $>$ SO $<$ sub $>16</$ sub $>$

Research articleAbstract only

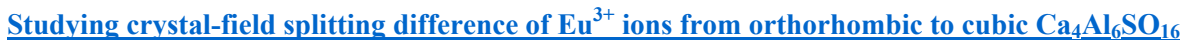

Jinpu Zhang, Jiaming Wu, Xiaolei Lu, Shuwei Ma, ... Xin Cheng

Pages 5998-6005

$\Perp_{\text {View PDF }}$

Article preview ${ }^{\vee}$

55. $\square$ select article Electrospun composite nanofibers for intermediate-temperature solid oxide fuel cell electrodes

Research articleAbstract only

Electrospun composite nanofibers for intermediate-temperature solid oxide fuel cell electrodes

Minwoo Ahn, Seungwoo Han, Jongseo Lee, Wonyoung Lee

Pages 6006-6011

歨 View PDF

Article preview ${ }^{\vee}$ 
56. $\square$ select article Novel direct $\mathrm{Z}$-scheme $\mathrm{AgI} / \mathrm{N}-\mathrm{TiO}<\mathrm{sub}>2</ \mathrm{sub}>$ photocatalyst for removal of polluted tetracycline under visible irradiation Research articleAbstract only

Novel direct Z-scheme $\mathrm{AgI} / \mathrm{N}-\mathrm{TiO}_{2}{ }_{2}$ photocatalyst for removal of polluted tetracycline under visible irradiation

Mai Hung Thanh Tung, Nguyen Thi Dieu Cam, Doan Van Thuan, Pham Van Quan, ... Thanh-Dong Pham Pages 6012-6021

עView PDF

Article preview $\vee$

57. $\square$ select article Transformation of metallic polymer precursor into nanosized $\mathrm{HfTaC}<\mathrm{sub}>2</$ sub $>$ ceramics

Research articleAbstract only

Transformation of metallic polymer precursor into nanosized $\mathrm{HfTaC}_{2}$ ceramics

Yanan Sun, Chunming Yang, Yan Lu, Fenghua Chen, ... Tong Zhao

Pages 6022-6028

$\boldsymbol{\Downarrow}_{\text {View PDF }}$

Article preview ${ }^{\vee}$

58. $\square$ select article A continuum damage mechanics model for 2-D woven oxide/oxide ceramic matrix composites under cyclic thermal shocks

Research articleAbstract only

A continuum damage mechanics model for 2-D woven oxide/oxide ceramic matrix composites under cyclic thermal shocks

Zhengmao Yang, Hui Lui
Pages 6029-6037
$\downarrow$ View PDF
Article preview $\checkmark$

59. $\square$ select article Laser surface modification of porous yttria stabilized zirconia against CMAS degradation

Research articleAbstract only

Laser surface modification of porous yttria stabilized zirconia against CMAS degradation

S. Bakkar, M.V. Pantawane, J.J. Gu, A. Ghoshal, ... S.M. Aouadi Pages 6038-6045

$\downarrow$ View PDF

Article preview ${ }^{\vee}$

60. $\square$ select article Gamma and neutron shielding characterizations of the Ag $<$ sub $>2</$ sub $>\mathrm{O}-\mathrm{V}<$ sub $>2</$ sub $>0<$ sub $>5</$ sub $>-\mathrm{MoO}<$ sub $>3</$ sub $>$ $-\mathrm{TeO}<\mathrm{sub}>2</$ sub $>$ quaternary tellurite glass system with the Geant 4 simulation toolkit and Phy-X software

Research articleAbstract only

Gamma and neutron shielding characterizations of the $\mathrm{Ag}_{2} \mathrm{O}-\mathrm{V}_{2} \mathrm{O}_{5}-\mathrm{MoO}_{3}-\mathrm{TeO}_{2}$ quaternary tellurite glass system with the Geant4 simulation toolkit and Phy-X software

A. Așkın

Pages 6046-6051

עView PDF

Article preview ${ }^{\vee}$

61. $\square$ select article Synthesis and morphology optimization of electrospun SiBNC nanofibers

Research articleAbstract only

Synthesis and morphology optimization of electrospun SiBNC nanofibers

Kamal Asadi-Pakdel, Rouhollah Mehdinavaz Aghdam, Mehdi Shahedi Asl, Mohammad Ali Faghihi Sani

Pages 6052-6059

$\boldsymbol{w}_{\text {View PDF }}$

Article preview $\vee$

62. $\square$ select article Characterization on the yield stress and interfacial coefficient of friction of glasses from scratch tests

Research articleAbstract only

Characterization on the yield stress and interfacial coefficient of friction of glasses from scratch tests

HaiTao Liu, MingHao Zhao, Chunsheng Lu, JianWei Zhang

Pages 6060-6066 
$\stackrel{\Downarrow \text { View PDF }}{\underline{4}}$

Article preview ${ }^{\vee}$

63. $\square$ select article Toward oxygen fully stoichiometric $\mathrm{La}<$ sub $>1-\mathrm{x}</$ sub $>\operatorname{Sr}<$ sub $>\mathrm{x}</$ sub $>\operatorname{CoO}<$ sub $>3</$ sub $>(0.5 \leq \mathrm{x} \leq 0.9)$ perovskites: Itinerant magnetic mechanism more than double exchange one's

Research articleAbstract only

Toward oxygen fully stoichiometric $\mathrm{La}_{1-\mathrm{x}} \mathrm{Sr}_{\mathrm{x}} \mathrm{CoO}_{3}(0.5 \leq \mathrm{x} \leq 0.9)$ perovskites: Itinerant magnetic mechanism more than double exchange one's

Madhu Chennabasappa, Emmauel Petit, Olivier Toulemonde

Pages 6067-6072

$\boldsymbol{\omega}_{\text {View PDF }}$

Article preview ${ }^{\vee}$

64. $\square$ select article Modified structural, optical, magnetic and ferroelectric properties in $(1-<\mathrm{em}>\mathrm{x}</ \mathrm{em}>)$

$\mathrm{BaTiO}<\mathrm{sub}>3</$ sub $>-<\mathrm{em}>\mathrm{x}</ \mathrm{em}>\mathrm{BaCo}<\mathrm{sub}>0.5</$ sub $>\mathrm{Nb}<\mathrm{sub}>0.5</$ sub $>0<$ sub $>3-\delta</$ sub $>$ ceramics

Research articleAbstract only

Modified structural, optical, magnetic and ferroelectric properties in $(1-x) \mathrm{BaTiO}_{3}-x \mathrm{BaCo}_{0.5} \underline{\mathrm{Nb}}_{0.5} \underline{\mathrm{O}}_{3-\delta} \underline{\text { ceramics }}$

Dongliang Zheng, Hongmei Deng, Shufang Si, Yanlin Pan, ... Junhao Chu

Pages 6073-6078

$\downarrow_{\text {View PDF }}$

Article preview ${ }^{\vee}$

65. $\square$ select article Novel temperature stable $\mathrm{NiSnTa}<\mathrm{sub}>2</$ sub $>\mathrm{O}<$ sub $>8</$ sub $>$ microwave dielectric ceramics with trirutile structure

Research articleAbstract only

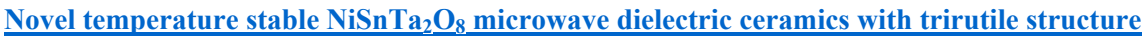

Enzhu Li, Qingyu Wen, Hongcheng Yang, Hongyu Yang, ... Shuren Zhang

Pages 6079-6084

$\boldsymbol{\iota}_{\text {View PDF }}$

Article preview ${ }^{\vee}$

66. $\square$ select article Synthesis of pyrochlore-borosilicate glass-ceramics for immobilization of high-level nuclear waste

Research articleAbstract only

Synthesis of pyrochlore-borosilicate glass-ceramics for immobilization of high-level nuclear waste

Kangming Wu, Fu Wang, Qilong Liao, Hanzhen Zhu, ... Yongchang Zhu

Pages 6085-6094

实 View PDF

Article preview ${ }^{\vee}$

67. $\square$ select article Green synthesis of dysprosium stannate nanoparticles using Ficus carica extract as photocatalyst for the degradation of organic pollutants under visible irradiation

Research articleAbstract only

Green synthesis of dysprosium stannate nanoparticles using Ficus carica extract as photocatalyst for the degradation of organic pollutants under visible irradiation

Sahar Zinatloo-Ajabshir, Maryam Sadat Morassaei, Omid Amiri, Masoud Salavati-Niasari

Pages 6095-6107

崖iew PDF

Article preview $\vee$

68. $\square$ select article Crystal structure, relaxor behaviors and energy storage performance of $(\mathrm{Sr}<\mathrm{sub}>0.7</$ sub $>\mathrm{Ba}<\mathrm{sub}>0.3</$ sub $>)$ $<$ sub $>5</$ sub $>$ LaNb $<$ sub $>7</$ sub $>$ Ti $<$ sub $>3</$ sub $>0<$ sub $>30</$ sub $>$ tungsten bronze ceramics

Research articleAbstract only

Crystal structure, relaxor behaviors and energy storage performance of ( $\left.\mathrm{Sr}_{0.7} \underline{\mathrm{Ba}}_{0.3}\right)_{5} \underline{\mathrm{LaNb}}_{7} \underline{\mathrm{Ti}}_{3} \underline{\mathrm{O}}_{30} \underline{\text { tungsten bronze ceramics }}$

Lei Cao, Ying Yuan, Zhengyi Yang, Enzhu Li, Shuren Zhang

Pages 6108-6114

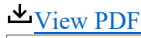

Article preview ${ }^{\vee}$ 
69. $\square$ select article Ultrasonic-assisted electrodeposition of $\mathrm{Ni}-\mathrm{Al}<\mathrm{sub}>2</$ sub $>\mathrm{O}<\mathrm{sub}>3</$ sub $>$ nanocomposites at various ultrasonic powers

Research articleAbstract only

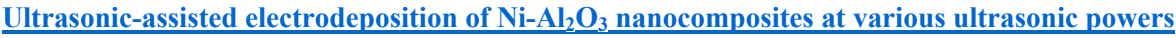

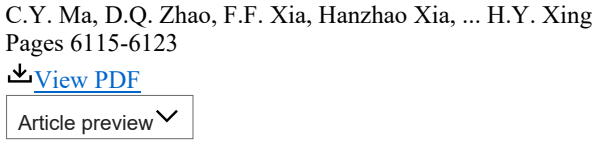

70. $\square$ select article Evaluation of GO nanosheets decorated by $\mathrm{CuFe}<$ sub $>2</$ sub $>\mathrm{O}<\mathrm{sub}>4</$ sub $>$ and CdS nanoparticles as photocatalyst for the degradation of dinoseb and imidacloprid pesticides

Research articleAbstract only

Evaluation of GO nanosheets decorated by $\mathrm{CuFe}_{2} \underline{\mathrm{O}}_{4}$ and CdS nanoparticles as photocatalyst for the degradation of dinoseb and imidacloprid pesticides

Mostafa Zangiabadi, Asma Saljooqi, Tayebeh Shamspur, Ali Mostafavi

Pages 6124-6128

$\underline{\text { View PDF }}$

Article preview $\vee$

71. $\square$ select article Design of functionalized $\alpha-\mathrm{Fe}<\mathrm{sub}>2</$ sub $>\mathrm{O}<$ sub $>3</$ sub $>$ (III) films with long-term anti-wetting properties

Research articleAbstract only

$\underline{\text { Design of functionalized } \alpha-\mathrm{Fe}_{2}} \underline{\mathrm{O}}_{3} \underline{\text { (III) films with long-term anti-wetting properties }}$

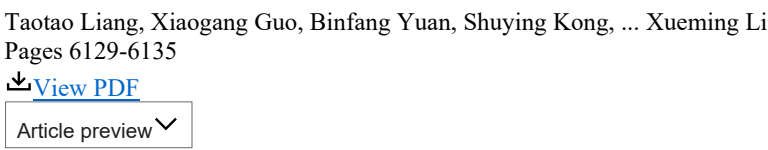

72. $\square$ select article A comprehensive study on the effect of TeO2 on the radiation shielding properties of $\mathrm{TeO}<$ sub $>2</$ sub $>-\mathrm{B}<$ sub $>2</$ sub $>\mathrm{O}<$ sub $>3</$ sub $>$ $-\mathrm{Bi}<\mathrm{sub}>2</$ sub $>\mathrm{O}<$ sub $>3</$ sub $>-\mathrm{LiF}-\mathrm{SrCl}<$ sub $>2</$ sub $>$ glass system using Phy-X $/$ PSD software

Research articleAbstract only

A comprehensive study on the effect of $\mathrm{TeO} 2$ on the radiation shielding properties of $\mathrm{TeO}_{2}-\mathrm{B}_{2} \underline{\mathrm{O}}_{3}-\mathrm{Bi}_{2} \mathrm{O}_{3}-\mathrm{LiF}-\mathrm{SrCl}{ }_{2}$ glass system using Phy-X / PSD software

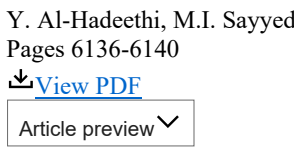

73. $\square$ select article Effect of $\mathrm{Nb}$ substitution on magnetic, ferroelectric and photocatalytic properties of $\mathrm{Bi}<$ sub $>0.95</$ sub $>$ Sm $<$ sub $>0.05</$ sub $>$ Fe $<$ sub $>1-<$ em $>$ x $</$ em $></$ sub $>$ Nb $<$ sub $><$ em $>$ X $</$ em $></$ sub $>0<$ sub $>3</$ sub $>(0 \leq<$ em $>$ x $</$ em $>\leq 0.1)$ nanoparticles Research articleAbstract only

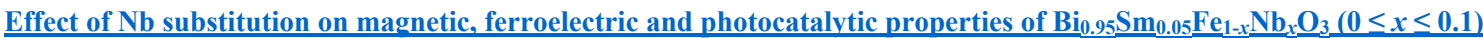
nanoparticles

F.L. Wang, Y. Li, N. Wang, Aditya Jain, ... F.G. Chen

Pages 6141-6145

实View PDF

Article preview ${ }^{\vee}$

74. $\square$ select article Preparation of spherical and porous strontium titanate particles by hot water and hydrothermal conversion of hydrous titania

Research articleAbstract only

Preparation of spherical and porous strontium titanate particles by hot water and hydrothermal conversion of hydrous titania

Kazuya Ujiie, Takashi Kojima, Kosuke Ota, Pornjira Phuenhinlad, ... Naofumi Uekawa

Pages 6146-6153

$\Perp_{\text {View PDF }}$

Article preview ${ }^{\vee}$

75. $\square$ select article Luminescence and self-referenced optical temperature sensing performance in $\mathrm{Ca}<$ sub $>2</$ sub $>\mathrm{YZr}<$ sub $>2</$ sub $>$ Al $<$ sub $>3</$ sub $>\mathrm{O}<$ sub $>12</$ sub $>$ :Bi $<$ sup $>3+</$ sup $>$,Eu $<$ sup $>3+</$ sup $>$ phosphor

Research articleAbstract only 
Luminescence and self-referenced optical temperature sensing performance in $\mathrm{Ca}_{2} \underline{\mathrm{YZr}}_{2} \underline{\mathrm{Al}}_{3} \underline{\mathrm{O}}_{12}: \mathrm{Bi}^{3+}{ }_{,} \mathrm{Eu}^{3+}{ }_{\text {phosphors }}$

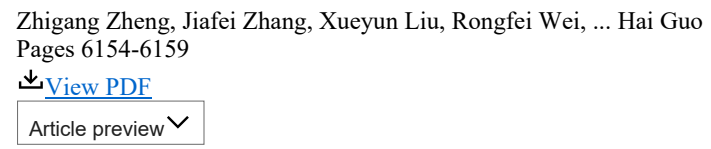

76. $\square$ select article Phase relations in the " $\mathrm{FeO}-\mathrm{V}<\mathrm{sub}>2</$ sub $>\mathrm{O}<$ sub $>3</$ sub $>$ " system at $1473 \mathrm{~K}$ and the magnetic properties of spinel phase $\mathrm{Fe}<$ sub $>3-<$ em $>\mathrm{x}</$ em $></$ sub $>\mathrm{V}<$ sub $><$ em $>\mathrm{x}</$ em $></$ sub $>\mathrm{O}<$ sub $>4</$ sub $>$

Research articleAbstract only

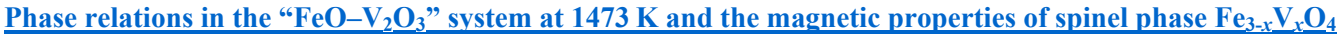

Baijun Yan, Daya Wang, Qiugen Qiu, Tengfei Deng Pages 6160-6167

$\downarrow_{\text {View PDF }}$

Article preview ${ }^{\vee}$

77. $\square$ select article High temperature microwave absorbing properties of plasma sprayed

$\mathrm{La}<$ sub $>0.6</$ sub $>\mathrm{Sr}<$ sub $>0.4</$ sub $>\mathrm{FeO}<$ sub $>3</$ sub $>-<$ sub $>\delta</$ sub $>/ \mathrm{MgAl}<$ sub $>2</$ sub $>\mathrm{O}<$ sub $>4</$ sub $>$ composite ceramic coatings Research articleAbstract only

High temperature microwave absorbing properties of plasma sprayed $\mathrm{La}_{0.6} \underline{\mathrm{Sr}}_{0.4} \underline{\mathrm{FeO}}_{3} \underline{-}_{\delta} / \underline{\mathrm{MgAl}}_{2} \underline{\mathrm{O}}_{4} \underline{\text { composite }}_{2}$ ceramic coatings

Hongyao Jia, Wancheng Zhou, Hanyi Nan, Yuchang Qing, ... Dongmei Zhu Pages 6168-6173

עView PDF

Article preview ${ }^{\vee}$

78. $\square$ select article Impact of $\mathrm{ZrO}<\mathrm{sub}>2</$ sub $>$ alloying on thermo-mechanical properties of $\mathrm{Gd}<\mathrm{sub}>3</$ sub $>\mathrm{NbO}<$ sub $>7</$ sub $>$

Research articleAbstract only

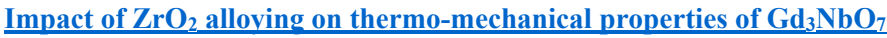

YiTao Wang, Lin Chen, Jing Feng Pages 6174-6181

山View PDF

Article preview $\vee$

79. $\square$ select article Effect of additives on slip casting rheology, microstructure and mechanical properties of $\mathrm{Si}<$ sub $>3</$ sub $>\mathrm{N}<$ sub $>4</$ sub $>/ \mathrm{SiC}$ composites Research articleAbstract only

Effect of additives on slip casting rheology, microstructure and mechanical properties of $\mathrm{Si}_{3} \underline{N}_{4} / \mathrm{SiC}_{\text {composites }}$

Shohreh Shahrestani, Mokhtar Che Ismail, Saeid Kakooei, Mohammadali Beheshti Pages 6182-6190

$\boldsymbol{\iota}_{\text {View PDF }}$

Article preview $\vee$

80. $\square$ select article Composite fibers of $\mathrm{La}<\mathrm{sub}>0.5</$ sub $>\mathrm{Sr}<\mathrm{sub}>0.5</$ sub $>\mathrm{CoO}<$ sub $>3-<$ em $>\delta</$ em $></$ sub $>$-LaSrCoO $<$ sub $>4 \pm<$ em $>\delta</$ em $></$ sub $>$ with high catalytic activity toward oxygen reduction reaction

Research articleAbstract only

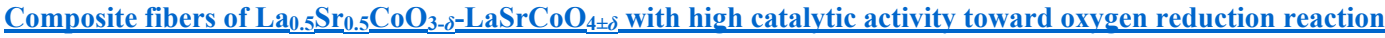

Fushao Li, Yingxian Xu, Feixiang Cheng, Yuxing Yan, ... Long Jiang Pages 6191-6198

步

Article preview $\vee$

81. $\square$ select article $2 \mathrm{D} \mathrm{Ti}<$ sub $>3</$ sub $>\mathrm{C}<$ sub $>2</$ sub $>\mathrm{T}<\mathrm{sub}>\mathrm{x}</$ sub $>$ MXene/aramid nanofibers composite films prepared via a simple filtration method with excellent mechanical and electromagnetic interference shielding properties

Research articleAbstract only

$\underline{2 D} \mathrm{Ti}_{3} \underline{\mathrm{C}}_{2} \underline{\mathrm{T}}_{\mathrm{x}}$ MXene/aramid nanofibers composite films prepared via a simple filtration method with excellent mechanical and electromagnetic interference shielding properties

Huawei Wei, Mingqiang Wang, Wenhui Zheng, Zaixing Jiang, Yudong Huang

Pages 6199-6204

עView PDF 
Article preview ${ }^{\vee}$

82. $\square$ select article Effect of varying $\mathrm{Al}<$ sub $>2</$ sub $>\mathrm{O}<$ sub $>3</$ sub $>$ contents of $\mathrm{CaO}-\mathrm{Al}<$ sub $>2</$ sub $>\mathrm{O}<$ sub $>3</$ sub $>-\mathrm{SiO}<$ sub $>2</$ sub $>$ slags on lumped $\mathrm{MgO}$ dissolution

Research articleAbstract only

$\underline{\text { Effect of varying } \mathrm{Al}_{2} \underline{\mathrm{O}}_{3} \text { contents of } \mathrm{CaO}-\mathrm{Al}_{2} \underline{\mathrm{O}}_{3}-\mathrm{SiO}_{2} \text { slags on lumped } \mathrm{MgO} \text { dissolution }}$

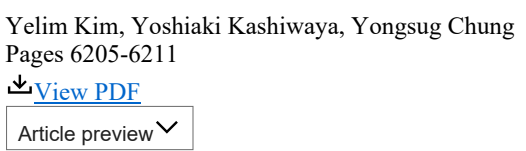

83. $\square$ select article A novel piezoelectric ceramic with high Curie temperature and high piezoelectric coefficient

Research articleAbstract only

$\underline{\text { A novel piezoelectric ceramic with high Curie temperature and high piezoelectric coefficient }}$

Siyu Huang, Jiangtao Zeng, Liaoying Zheng, Zhenyong Man, ... Guorong Li

Pages 6212-6216

责iew PDF

Article preview $\checkmark$

84. $\square$ select article Synthesis of $\mathrm{LiBaZrO}<\mathrm{sub}>\mathrm{x}</$ sub $>$ ceramics with a core-shell structure

Research articleAbstract only

$\underline{\text { Synthesis of } \mathrm{LiBaZrO}}{ }_{\mathrm{x}}$ ceramics with a core-shell structure

M.V. Zdorovets, A. Arbuz, A.L. Kozlovskiy

Pages 6217-6221

$\boldsymbol{\downarrow}_{\mathrm{View}}$ PDF

Article preview ${ }^{\vee}$

85. $\square$ select article Hydrothermal synthesis and characterization studies of $\alpha$-Fe $<$ sub $>2</$ sub $>\mathrm{O}<$ sub $>3</$ sub $>/ \mathrm{MnO}<$ sub $>2</$ sub $>$ nanocomposites for energy storage supercapacitor application

Research articleAbstract only

Hydrothermal synthesis and characterization studies of $\alpha-\mathrm{Fe}_{2} \mathrm{O}_{3} / \mathrm{MnO}_{2}$ nanocomposites for energy storage supercapacitor application

Mohamed Racik K, A. Manikandan, M. Mahendiran, J. Madhavan, ... T. Maiyalagan

Pages 6222-6233

$\boldsymbol{\omega}_{\text {View PDF }}$

Article preview ${ }^{\vee}$

86. $\square$ select article 3D-SiC decorated with SiC whiskers: Chemical vapor infiltration on the porous 3D-SiC lattices derived from polycarbosilane-based suspensions

Research articleAbstract only

3D-SiC decorated with $\mathrm{SiC}$ whiskers: Chemical vapor infiltration on the porous 3D-SiC lattices derived from polycarbosilanebased suspensions

Huiwen Xiong, Hehao Chen, Zhaoke Chen, Xiang Xiong, ... Kechao Zhou

Pages 6234-6242

歨 View PDF

Article preview $\vee$

87. $\square$ select article Nanomechanical characterization of RB-SiC ceramics based on nanoindentation and modelling of the ground surface roughness

Research articleAbstract only

Nanomechanical characterization of RB-SiC ceramics based on nanoindentation and modelling of the ground surface roughness

Zhenzhong Zhang, Peng Yao, Jun Wang, Chuanzhen Huang, ... Bin Zou

Pages 6243-6253

עView PDF

Article preview ${ }^{\vee}$

88. $\square$ select article Oxidation and cracking/spallation resistance of $\mathrm{ZrB}<$ sub $>2</$ sub $>-\mathrm{SiC}-\mathrm{TaSi}<$ sub $>2</$ sub $>-\mathrm{Si}$ coating on siliconized graphite at $1500{ }^{\circ} \mathrm{C}$ in air Research articleAbstract only 
$\underline{\text { Oxidation and cracking/spallation resistance of } \mathrm{ZrB}_{2}-\mathrm{SiC}_{2}-\mathrm{TaSi}_{2}-\mathrm{Si} \text { coating on siliconized graphite at } 1500{ }^{\circ} \mathrm{C} \text { in air }}$

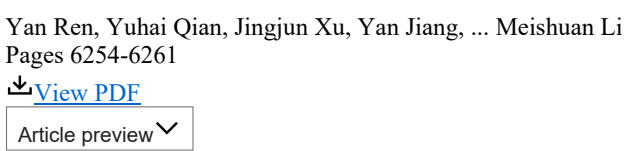

89. $\square$ select article Robust assembly of urchin-like $\mathrm{NiCo}<\mathrm{sub}>2</$ sub $>\mathrm{O}<$ sub $>4</$ sub $>/$ CNTs architecture as bifunctional electrocatalyst in $\mathrm{Zn}$-Air batteries Research articleAbstract only

$\underline{\text { Robust assembly of urchin-like } \mathrm{NiC}_{2}} \underline{\mathrm{O}}_{4} / \mathrm{CNTs}$ architecture as bifunctional electrocatalyst in $\mathrm{Zn}$-Air batteries

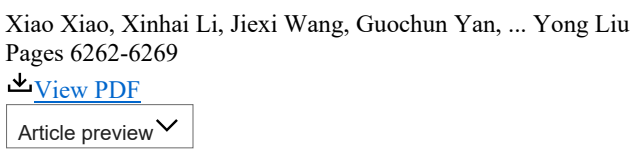

90. $\square$ select article Thermo-mechanical behaviours of $\mathrm{Li}<\mathrm{sub}>3<\mathrm{em}>\mathrm{x}</ \mathrm{em}></$ sub $>$ La $<$ sub $>1 / 3-<\mathrm{em}>\mathrm{x}</$ em $></$ sub $>\mathrm{MO}<$ sub $>3</$ sub $>(\mathrm{M}=\mathrm{Ta}$ and $\mathrm{Nb})$

Research articleAbstract only

Thermo-mechanical behaviours of $\mathrm{Li}_{3} x \underline{\mathrm{La}}_{1 / 3-x} \underline{\mathrm{MO}}_{3} \underline{(\mathrm{M}=\mathrm{Ta} \text { and } \mathrm{Nb})}$

Wakako Araki, Yasuhiro Nagakura, Yoshio Arai Pages 6270-6275

齿iew PDF

Article preview $\vee$

91. $\square$ select article Synthesis, energy transfer mechanism, and tunable emissions of novel $\mathrm{Na}<\mathrm{sub}>3</$ sub $>\mathrm{La}(\mathrm{VO}<$ sub $>4</$ sub $>$ ) $<$ sub $>2</$ sub $>$ : $\operatorname{Re}<$ sup $>3+</$ sup $>(\operatorname{Re}<$ sup $>3+</$ sup $>=$ Dy $<$ sup $>3+</$ sup $>$, Eu $<$ sup $>3+</$ sup $>$, and Sm $<$ sup $>3+</$ sup $>)$ vanadate phosphors for near-UVexcited white LEDs

Research articleAbstract only

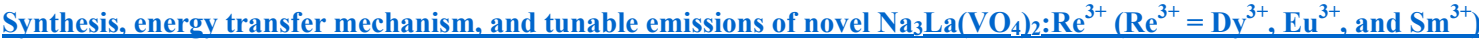
vanadate phosphors for near-UV-excited white LEDs

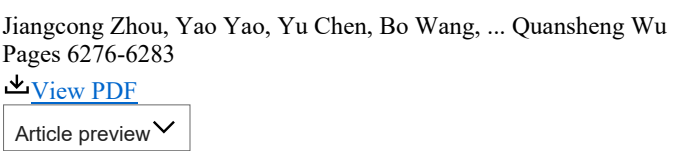

92. $\square$ select article Photocatalytic activity of copper ferrite graphene oxide particles for an efficient catalytic degradation of Reactive Black 5 in water

Research articleAbstract only

Photocatalytic activity of copper ferrite graphene oxide particles for an efficient catalytic degradation of Reactive Black 5 in water

Rasmus Kodasma, Burcu Palas, Gülin Ersöz, Süheyda Atalay Pages 6284-6292

$\Perp_{\text {View PDF }}$

Article preview ${ }^{\top}$

93. $\square$ select article Anomalous thermal expansion behavior in $\mathrm{Er}<$ sub $>1-<$ em $>\mathrm{x}</$ em $></$ sub $>\mathrm{Ca}<$ sub $><$ em $>$ x $</$ em $></$ sub $>$ MnO $<$ sub $>3</$ sub $>$

Research articleAbstract only

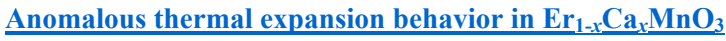

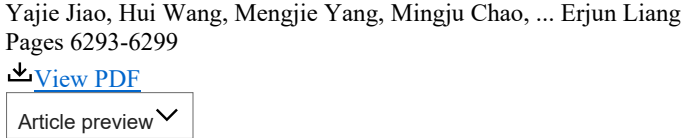

94. $\square$ select article $\mathrm{Ti}(\mathrm{C}, \mathrm{N})$-based cermets with fine grains and uniformly dispersed binders: Effect of the Ni-Co based binders

Research articleAbstract only

$\underline{\mathrm{Ti}(\mathrm{C}, \mathrm{N}) \text {-based cermets with fine grains and uniformly dispersed binders: Effect of the Ni-Co based binders }}$

Dingqian Dong, Wei Yang, Huiwen Xiong, Li Zhang, ... Jun Liao Pages 6300-6310

${ }_{\text {View PDF }}$ 
Article preview ${ }^{\vee}$

95. $\square$ select article Characteristics of Optimized Al:ZnO sputtering targets prepared by nanostructured powder

Research articleAbstract only

Characteristics of Optimized $\mathrm{Al}: \mathrm{ZnO}$ sputtering targets prepared by nanostructured powder

Haichao Liu, Ming Gao, Benrun Yang, Haojie Chen, ... Hu Zhang

Pages 6311-6317

$\boldsymbol{\Perp}_{\text {View PDF }}$

Article preview ${ }^{\vee}$

96. $\square$ select article Optical, structural, morphological, and compositional properties of cobalt doped tin oxide (CTO) thin films deposited by modified chemical bath method in alkaline medium

Research articleAbstract only

Optical, structural, morphological, and compositional properties of cobalt doped tin oxide (CTO) thin films deposited by modified chemical bath method in alkaline medium

L.N. Ezenwaka, N.S. Umeokwonna, N.L. Okoli

Pages 6318-6325

实iew PDF

Article preview ${ }^{\vee}$

97. $\square$ select article Preparation and characterization of organic-inorganic hybrid ZrOC/PF aerogel used as high-temperature insulator

Research articleAbstract only

Preparation and characterization of organic-inorganic hybrid ZrOC/PF aerogel used as high-temperature insulator

Sue Ren, li Hu, Xiutao Li, Jinpeng Fan, Jun Liang

Pages 6326-6332

崖iew PDF

Article preview ${ }^{\vee}$

K $\langle$ Previous page 1 of 2 Next $\rangle \mid$

<Previous vol/issue

Next vol/issue

ISSN: 0272-8842

Copyright (C) 2020 Elsevier Ltd and Techna S.r.l. All rights reserved.

\section{Else}

vier
logo

$$
\begin{aligned}
& \frac{\text { About ScienceDirect }}{\text { Remote access }} \\
& \frac{\text { Shopping cart }}{\text { Advertise }} \\
& \hline \text { Contact and support } \\
& \text { Terms and conditions } \\
& \text { Privacy policy }
\end{aligned}
$$

We use cookies to help provide and enhance our service and tailor content and ads. By continuing you agree to the use of cookies

Copyright (C) 2020 Elsevier B.V. or its licensors or contributors. ScienceDirect ${ }^{\circledR}$ is a registered trademark of Elsevier B.V.

ScienceDirect ${ }^{\circledR}$ is a registered trademark of Elsevier B.V.

QRELX'M 


\title{
Size controlled synthesis of well-distributed nano-silver on hydroxyapatite using alkanolamine compounds
}

\author{
Arniati Labanni ${ }^{\mathrm{a}}$, Zulhadjri ${ }^{\mathrm{a}}$, Dian Handayani ${ }^{\mathrm{b}}$, Yutaka Ohya ${ }^{\mathrm{c}}$, Syukri Arief ${ }^{\mathrm{a}, *}$ \\ ${ }^{a}$ Department of Chemistry, Faculty of Mathematics and Sciences, Andalas University, Padang, 25163, Indonesia \\ ${ }^{\mathrm{b}}$ Faculty of Pharmacy, Andalas University, Padang, 25163, Indonesia \\ ${ }^{\mathrm{c}}$ Department of Chemistry and Biomolecular Science, Faculty of Engineering, Gifu University, Yanagido 1 - 1, Gifu, 501-1193, Japan
}

\section{A R T I C L E I N F O}

\section{Keywords:}

Silver nanoparticle

Silver hydroxyapatite composite

Uncaria gambir Roxb.

Bone implant

\begin{abstract}
A B S T R A C T
A well-distributed nano-silver hydroxyapatite composite has been successfully prepared by a one-pot synthesis method. Hydroxyapatite was separately synthesized by a sol-gel method, then impregnated with silver nanoparticles with the mediation of Uncaria gambir Roxb. leaf extract in the presence of three kinds of alkanolamine compound; monoethanolamine (MEA), diethanolamine (DEA), and triethanolamine (TEA) as capping agents. The effect of different capping agents on the properties of the silver nanoparticles and the nano-silver hydroxyapatite composite were studied. UV-visible spectrophotometer analysis exhibited absorbance peaks at 402-439 nm which specifically corresponds to spherical silver nanoparticles. Higher optical absorbance was observed in TEA-capped silver nanoparticles, than in DEA and MEA-capped ones. X-ray diffraction (XRD) analysis showed a highly crystalline hexagonal structure for hydroxyapatite and no detected metallic silver. However, the presence of $1.65 \%$ silver was confirmed by energy dispersive x-ray (EDX) spectroscopy analysis. Transmission electron microscopy (TEM) analysis revealed spherical silver nanoparticles with a size range of 2-62 $\mathrm{nm}$ (smallest mean diameter of $2 \mathrm{~nm}$ ) adhered to the hydroxyapatite surface. The TEA capped impregnated silver nanoparticles were the smallest, corresponding to the best capping performance, followed by those capped by DEA and MEA. Small-sized nanoparticles on hydroxyapatite are beneficial for highly antibacterial bone implants.
\end{abstract}

\section{Introduction}

In recent decades, the study of calcium orthophosphate based inorganic biomaterials has been of increasing interest due to their wide applications in the field of health and medicine [1-3]. One of the most studied of these is hydroxyapatite (HA) (chemical formula $\mathrm{Ca}_{10}\left(\mathrm{PO}_{4}\right)_{6}(\mathrm{OH})_{2}$ ) which is used as a graft material for bone and for tooth substitution and repair due to its biocompatibility, bioactivity, and osteoconductivity [4,5]. Synthesis of hydroxyapatite has been achieved using a hydrothermal method [6], a microwave-assisted method [1], sol-gel [2], and a precipitation method [5]. The sol-gel method is considered efficient, easy, and environmentally friendly as it is low-cost, requires a low formation temperature and yet achieves high degrees of homogeneity and purity [7].

However, one of the main problems of this material is infections that can occur after bone implantation or other surgical operations since these biomaterials provide sites for potential bacteria adhesion. This process might be due to the absorption of protein, amino acid, and any other organic compounds into HA [8]. Furthermore, antibiotics loaded into implant material tend to be rapidly flushed out by body flood resulting in long term post-surgical infections [9]. This situation is made worse by the increase of multi-drug resistant (MDR) bacteria. Therefore, there is a demand for the development of a new generation of modified antibacterial agents to solve this problem [10,11]. Nanotechnology offers a promising approach to the fabrication of antibacterial agents in the form of metallic nanoparticles. In recent years, silver nanoparticles with their antibacterial properties have gained a lot of attention due to their potential applications in the biomedical field $[12,13]$. It could be expected that the incorporation of silver nanoparticles into hydroxyapatite could help avoid post-operative inflammation and bacterial infection [14]. In addition, compared to bulk materials, silver nanoparticles have excellent properties due to the high surface area to volume ratio which results in a beneficial effect at low concentrations when incorporated into a biomaterial. This point is crucial for the health of the patient as it minimizes the amount of this metal in the body [15].

\footnotetext{
${ }^{*}$ Corresponding author.

E-mail address: syukriarief@sci.unand.ac.id (S. Arief).
} 
In our previous studies, stable colloidal silver nanoparticles have been successfully synthesized by bio-reducing $\mathrm{Ag}^{+}$using Uncaria gambir Roxb. as a reducing agent in presence of MEA [16] and DEA [17] as a capping agent. Uncaria gambir Roxb. is one of commodity in West Sumatera, Indonesia and for long time had been used as traditional medicine due to the content of some polyphenol compounds predominantly catechin. Those polyphenol compound is expected to reduce $\mathrm{Ag}^{+}$to $\mathrm{Ag}^{0}$. The formation of those stable and small-sized colloidal silver nanoparticles was conducted using a simple method and did not require the use of expensive technological equipment. Modifying this method of silver nanoparticle fabrication by the addition of a capping agent should result in control over their size and stability which would be advantageous since the antibacterial activity of metallic nanoparticles is strongly influenced by size [18]. Hence, in this study, three kinds of alkanolamine compound, i.e. MEA, DEA, and TEA were trialled as capping agents in the preparation of silver nanoparticles using Uncaria gambir Roxb. leaf extract using 1 pot synthesis with hydroxyapatite powder. The effect of alkanolamine compound structure on the properties of silver nanoparticles was studied and the effect of each capping agent were compared using UV-Vis spectrophotometry, XRD, EDX, and TEM analysis.

\section{Experimental procedures}

\subsection{Synthesis of hydroxyapatite}

Hydroxyapatite was separately synthesized using a sol-gel method based on previous report by Jamarun et al. [7]. The calcium precursor used in the reaction was extracted from cockle shells (Anadara granosa) as in Azis et al. (2015) [6]. The cockle shells were cleaned, washed, dried, and ground to a coarse powder. They were then calcined at $900{ }^{\circ} \mathrm{C}$ for $5 \mathrm{~h}$ to obtain $\mathrm{CaO}$. $\left(\mathrm{NH}_{4}\right)_{2} \mathrm{HPO}_{4}$ (Merck, analytical grade) was used as a phosphor precursor.

This $\mathrm{CaO}$ was diluted into $\mathrm{HNO}_{3} 2 \mathrm{M}$ (Merck, analytical grade) and then stirred at a constant rate of $600 \mathrm{rpm}$ at $85^{\circ} \mathrm{C}$ for $20 \mathrm{~min} 250 \mathrm{ml}$ $\left(\mathrm{NH}_{4}\right)_{2} \mathrm{HPO}_{4}$ was added drop-wise to the solution while stirring at $110^{\circ} \mathrm{C}$ and $\mathrm{pH}$ adjusted to 11 using $\mathrm{NH}_{4} \mathrm{OH}$ (Merck, Analytical grade). The mixture was stirred for $5 \mathrm{~h}$ to obtain white sol. After $24 \mathrm{~h}$ of aging, the gel was filtered and dried in a $110^{\circ} \mathrm{C}$ oven for $6 \mathrm{~h}$ to obtain a powder. This was then calcined at $900^{\circ} \mathrm{C}$ for $5 \mathrm{~h}$ resulting in hydroxyapatite.

\subsection{Synthesis of HAp-AgNps composite}

Uncaria gambir Roxb. leaves were taken from the experimental garden, Andalas University, Padang, Indonesia and extracted using the method of Labanni et al. [16]. Fresh leaves were washed, dehydrated by shade drying, and mashed up to obtain powder. Extraction was conducted using water solvent and stirring at a constant rate at $65^{\circ} \mathrm{C}$ for $2 \mathrm{~h}$ then filtered. This filtrate was then stored in a sealed bottle at $4^{\circ} \mathrm{C}$.

Nano-silver hydroxyapatite composite was fabricated by precipitation method. $1 \mathrm{mM}$ silver nitrate (Merck, Analytical grade), $2 \mathrm{mM}$ of the chosen alkanolamine (Merck, Analytical grade), and 4\% leaf extract with as-synthesized hydroxyapatite to make a total volume of $50 \mathrm{~mL}$ were stirred together in a dark environment in 1 pot for $24 \mathrm{~h}$ at a constant rate of $600 \mathrm{rpm}$ at room temperature. After stirring, the mixture was filtered and the residue was washed using distilled water, then dried in a hot air oven to produce powder. The synthesized nano-silver hydroxyapatite composite fabricated using MEA, DEA, or TEA as a capping agent are hereafter denoted as HAp-AgNps MEA, HAp-AgNps DEA, and HAp-AgNps TEA, respectively. In addition, the synthesis of HAp-AgNps without the use of a capping agent was also carried out as control, which is hereafter denoted as HAp-AgNps0.

\subsection{Characterization}

The UV-Vis Spectrophotometry analysis of silver nanoparticles was carried out using Thermo Scientific Evolution $201 \mathrm{UV}-\mathrm{Vis}$ in wavelength range of $200-800 \mathrm{~nm}$. The crystal structure of synthesized HApAgNps was investigated using X-Ray Diffraction Rigaku Ultima IV with CuKa radiation with a scanning rate of $2^{\circ} \mathrm{min}^{-1}$ at $60 \mathrm{kV}$ and $20 \mathrm{~mA}$. The size and the morphology of HAp-AgNps were evaluated using JEOL JEM 2100. Chemical composition of the samples was determined using EDX JEOL JED 2300.

\section{Results and discussion}

\subsection{UV-Vis Spectrophotometry analysis}

The formation of silver nanoparticles was indicated by a colour change from colourless to yellowish-brown $5 \mathrm{~min}$ after the reaction. The presence of the particles was then tested by UV-Vis spectrophotometry analysis over the wavelength range of $200-800 \mathrm{~nm}$ (Fig. 1). Optical absorption peaks occurring at $402-439 \mathrm{~nm}$ were observed in all four samples. The absorbance value increased with the presence of alkanolamine in the reaction which suggested that, besides acting as a capping agent as expected, these alkanolamine compounds acted as reducing agents as well. AgNps-TEA provided the highest absorbance value followed by AgNps-DEA, then AgNps-MEA. This result suggested that the structure of alkanolamine compounds used significantly affects the number of silver nanoparticles formed in the reaction. This might be due to the number of hydroxyl groups in the structure, which have a role in reducing silver cations to metallic silver. Furthermore, the use of different alkanolamine compounds also resulted in different wavelength ranges in the absorbance spectrum, with AgNps-TEA absorbing at shorter wavelength than AgNps-DEA and AgNps-MEA. The Mie theory of scattering for spherical metal nanoparticles indicates that a shift to longer wavelength corresponds to a larger particle size [19]. Hence, this result suggests that the use of TEA resulted in smaller sized nanoparticles than those of DEA and MEA capped reactions. AgNps-TEA capped nanoparticles also had a narrower absorbance peak suggesting a narrower size range. This result was subsequently affirmed by TEM analysis.

\subsection{X-ray diffraction analysis}

The result of XRD analysis of the HAp-AgNps powder synthesized with each alkanolamine compound is displayed in Fig. 2. The diffraction pattern of HAp-AgNps in all samples showed peaks at $2 \theta$ values of $26.10,32.00,32.43,33.16,46.86$, and $49.62^{\circ}$ indicating a well-

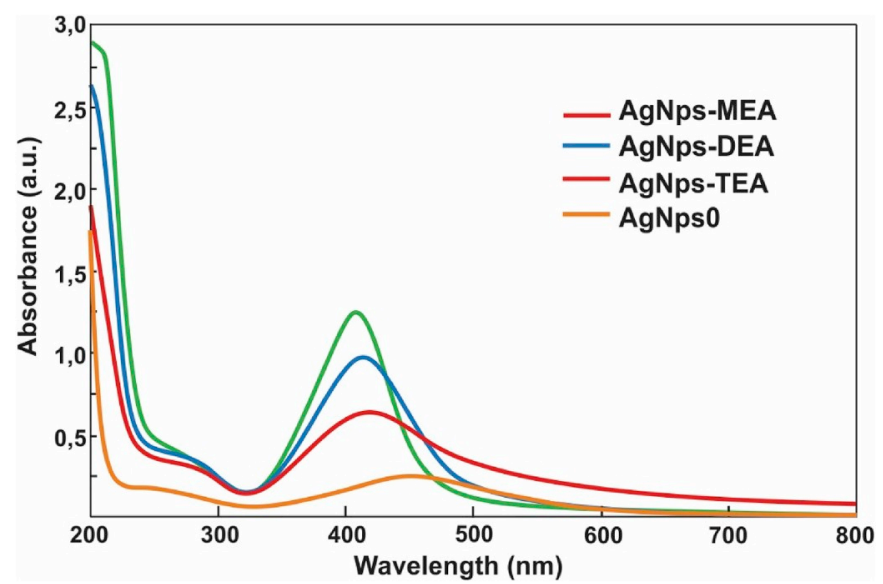

Fig. 1. UV-Vis absorption spectrum of Uncaria gambir Roxb. mediated silver nanoparticle with alkanolamine compounds as capping agent. 


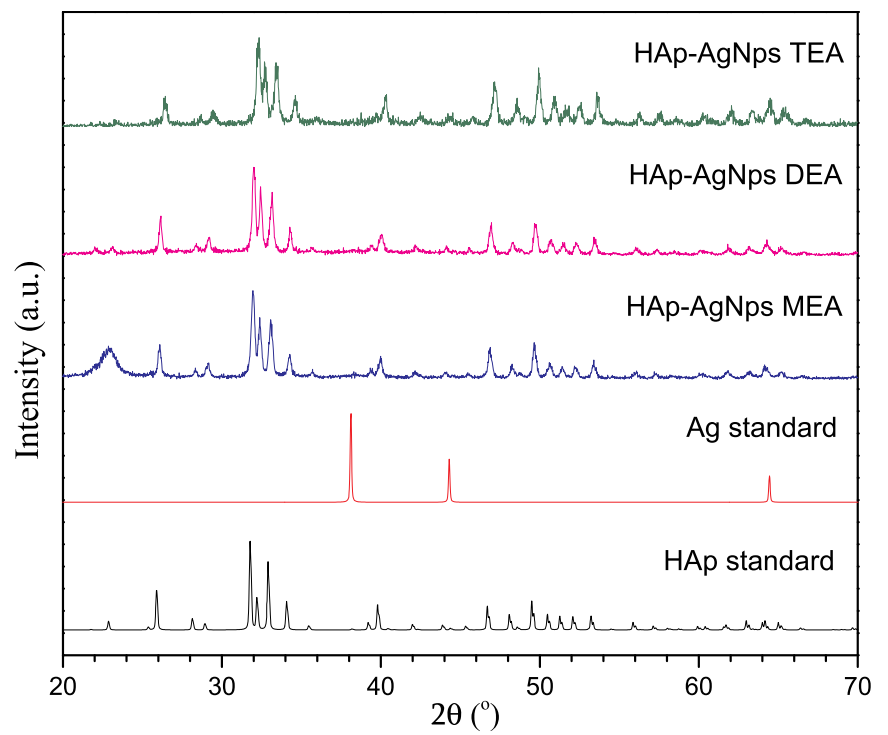

Fig. 2. XRD pattern of synthesized HAp-AgNps.

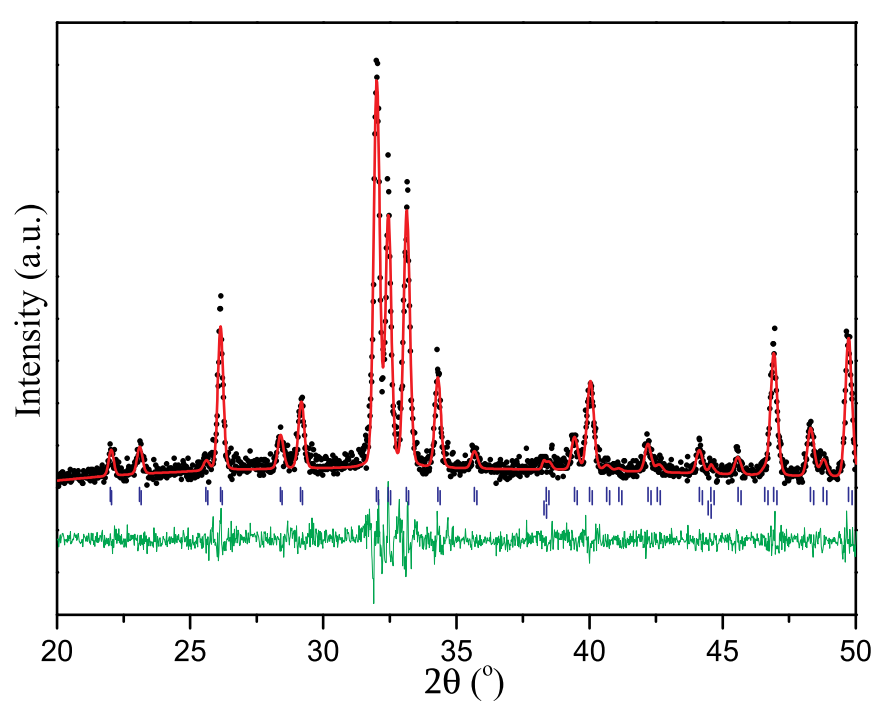

Fig. 3. XRD pattern fitted with Le Bail method for bi-phasic HAp-AgNps. crystallized hexagonal structured hydroxyapatite based on the ICSD \#26204 standard. This result indicated that hydroxyapatite had been successfully synthesized using Anadara granosa as a calcium precursor using sol-gel method. The metallic silver was not evident in the diffraction pattern of the HAp-AgNps samples. Similar results have also been found in previous researches about HAp-AgNps composite, where metallic silver was not detected in XRD analysis [20,21]. In fact, the percentage of silver nanoparticles was very small compared to hydroxyapatite in the samples and this could be the reason for the lack of observable metallic silver peaks.

In order to display the metallic silver peak in XRD analysis more clearly, Le Bail refinement was conducted on one of the samples using Rietica software using 2 phase standards of hydroxyapatite and silver. The plot is shown in Fig. 3, where the black circles represent the experimental XRD data, the red line represents calculated data of both hexagonal hydroxyapatite and face-centred cubic silver phases, and the green line represents the difference pattern. The result shows the presence of $\mathrm{Ag}$ at $2 \theta$ of 38.20 and $44.21^{\circ}$, marked by an asterisk (*), in accordance with the ICSD \#604631 standard for metallic silver. The Rp value was found to be 16.09 with a goodness of fit $\left(\chi^{2}\right)$ of 2.169 which is an acceptable value according to the basic principles of goodness of fit [22]. In comparison to this result, the $\mathrm{Rp}$ value of single-phase hydroxyapatite refinement was found to be 16.72 . The decrease of the Rp value in two-phase refinement indicates the presence of the $\mathrm{Ag}$ peak in the diffraction pattern.

\subsection{Energy dispersive X-Ray analysis}

Elemental analysis using EDX further confirmed the presence of $\mathrm{Ag}$ in the samples. Fig. 4 showed the elemental analysis result of HApAgNps TEA with an Ag peak at approximately $3 \mathrm{keV}$, with a mass percentage of $1.68 \%$. This clearly confirmed the presence of silver in the samples as indicated in the XRD analysis. Not surprisingly, Ca and $\mathrm{P}$ were also evident in the sample from the content of the hydroxyapatite.

\subsection{Transmission electron microscopy analysis}

The presence of nanometer-sized silver was clearly observed in the TEM. Spherical nanoparticles with size range of $2-15 \mathrm{~nm}$ could be clearly observed attached on the surface of micrometer-sized hydroxyapatite (Fig. 5). The very small size and ratio of silver nanoparticle compared to hydroxyapatite is a strong reason for the absence of metallic silver peaks in the XRD analysis result. A highly significant difference was observed between the appearance of the capped HAp-

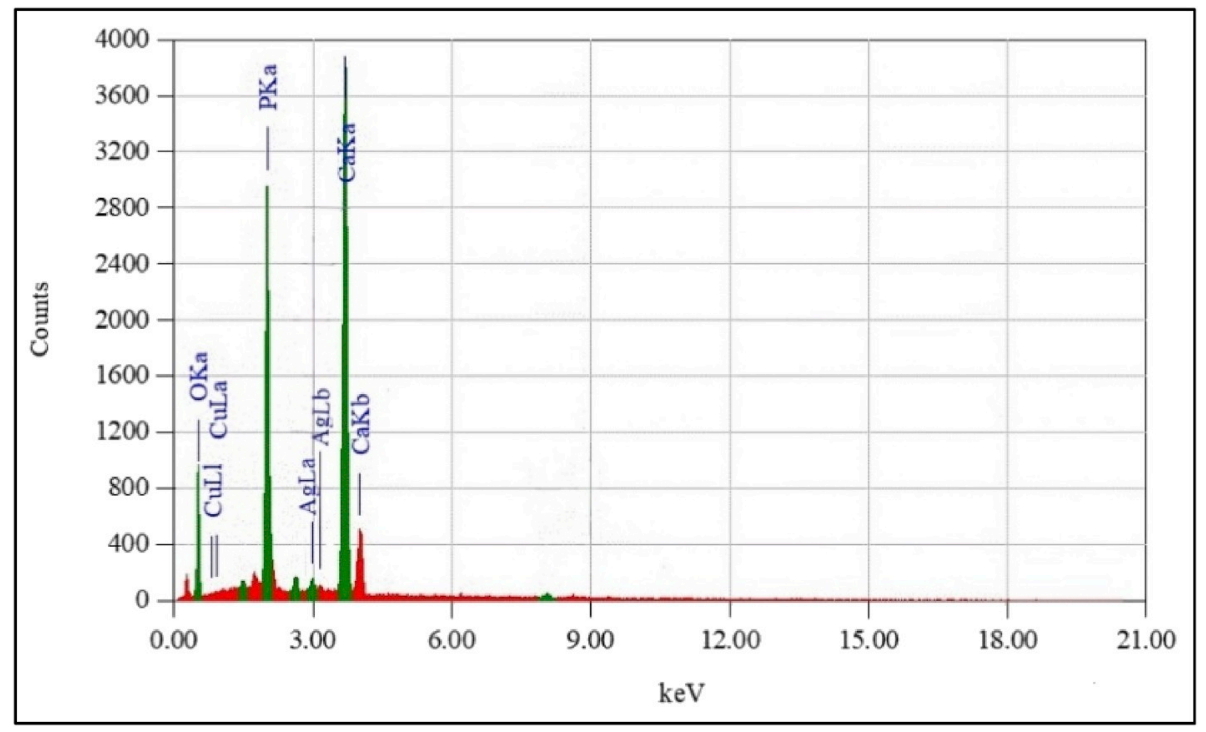

Fig. 4. EDX analysis of HAp-AgNps TEA. 

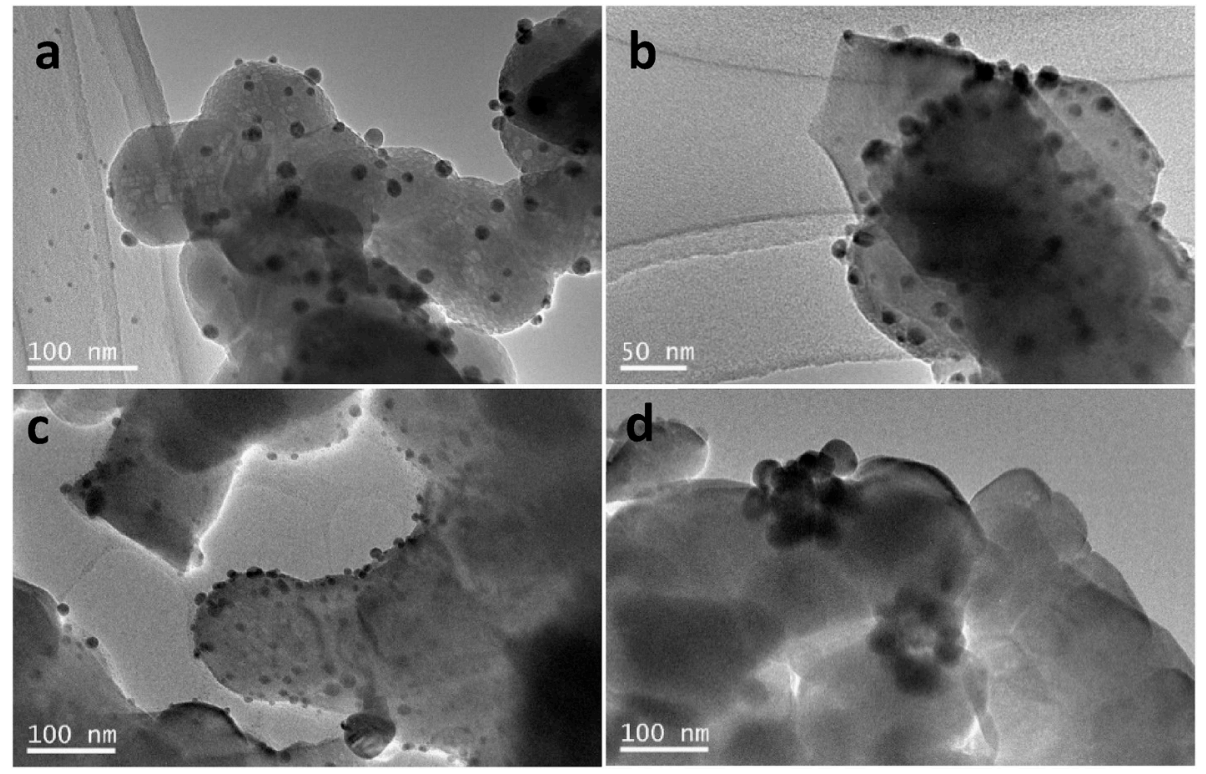

Fig. 5. TEM Images of a) HAp-AgNps MEA, b) HAp-AgNps DEA, c) HAp-AgNps TEA, and d) HAp-AgNps0.

AgNps and AgNps0. Silver nanoparticles on HAp-AgNps0 (Fig. 5d) tended to be stacked and agglomerated in certain sites, while in HAp AgNps-MEA, HAp-AgNps DEA, and HAp-AgNps TEA (Fig. 5a, b, c) silver nanoparticles were well distributed over the HAp surface. A similar result has been previously reported by Nirmala et al. [23] who synthesized HAp-AgNps from bovine femur bone where hydroxyapatite was presented as microstructure with silver nanoparticles attached on the periphery. The present results clearly suggest that the use of
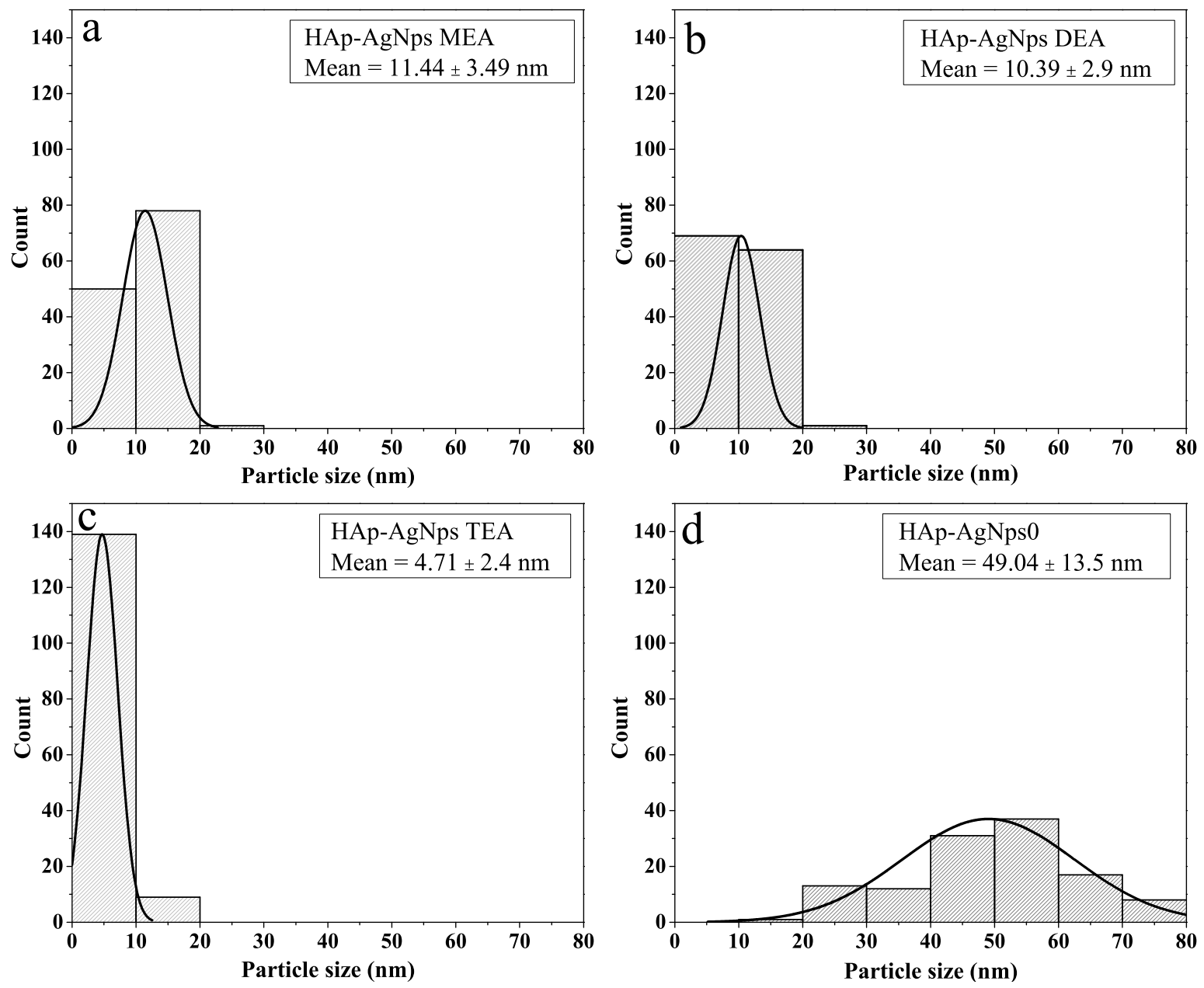

Fig. 6. Particle size distribution of AgNps on a) HAp-AgNps MEA, b) HAp-AgNps DEA, c) HAp-AgNps TEA, and d) HAp-AgNps0. 
alkanolamine compounds strongly affects the distribution of AgNps on the HAp surface. The attachment of silver nanoparticles on the surface of an implant is more advantageous for antibacterial activity than silver nanoparticles attached inside the implant [24].

Fig. 6 shows particle size distribution of as-synthesized HAp-AgNps as determined by ImageJ software. It was discovered that the mean diameter of nano-silver in HAp-AgNps0 was $49 \mathrm{~nm}, 10$ times larger than that of nano-silver in HAp-AgNps TEA. In addition, nano-silver in HApAgNps0 (Fig. 6d) showed a much broader particle size range than those in the alkanolamine capped samples indicating that the use of capping agents was indeed able to control growth and affect the size uniformity of nanoparticles.

In addition, there was a correlation between the type of capping agent used (MEA, DEA, and TEA) and the size of silver nanoparticles. Nano-silver in HAp-AgNps TEA provided the smallest particle size, followed by nano-silver in HAp-AgNps DEA, then HAp-AgNps MEA. As TEA has more hydroxyl groups than DEA and MEA has even fewer, it is concluded that the higher number of hydroxyl groups in the structure results in the smaller mean size. Several studies have reported that in the stabilizing mechanism of silver nanoparticles by alkanolamine compounds, an electrostatic interaction occurs between free electron pairs from nitrogen and positively charged AgNps, resulting in a protective monolayer. This monolayer then caps nanoparticles to avoid excessive fusion, hence controls the growth and size of nanoparticles $[16,25,26]$. It is assumed that hydroxyl groups of alkanolamine compound obstruct further interaction among silver nanoparticles. The higher number of hydroxyl groups in TEA could be the reason for the optimum capping performance of TEA, thus resulting in the smallest nanoparticle size seen in the TEM as well as the wavelength shift in the UV-Vis spectrophotometry analysis. These silver nanoparticles are of a much smaller size than those previously reported by Bharti et al. who used trisodium citrate as a reducing agent, resulting in nano-silver with mean particle size of $58.38 \mathrm{~nm}$ [27].

\section{Conclusion}

A well-distributed nano-silver hydroxyapatite composite has been successfully synthesized using a 1 pot green synthesis method with Uncaria gambir Roxb. leaf extract as the bio-reducing agent and each of three different alkanolamine compounds as capping agents. UV-Vis spectrophotometry and EDX analysis confirmed the formation of silver nanoparticles. XRD analysis showed the formation of a highly crystalline hexagonal structured hydroxyapatite. TEM analysis confirmed the presence of spherical silver nanoparticles with a diameter range of 2-62 $\mathrm{nm}$ adhering to the hydroxyapatite surface. The results suggested that alkanolamine compounds can play a crucial role in fabricating stable and well-distributed silver nanoparticles on the hydroxyapatite surface. HAp-AgNps TEA showed smaller AgNps than those of MEA and DEA. This small size indicates that although the amount of silver contained in the hydroxyapatite compound was extremely small, the silver's surface distribution should have a significant antibacterial impact. Silver nanoparticles incorporated hydroxyapatite using Uncaria gambir Roxb. leaf extract and TEA could produce materials highly suitable for biomedical applications.

\section{Declaration of competing interest}

The authors declare that they have no known competing financial interests or personal relationships that could have appeared to influence the work reported in this paper.

\section{Acknowledgement}

The authors would like to thank the Ministry of Research, Technology and Higher Education of the Republic of Indonesia and
LPPM of Andalas University for funding this work through the research grants of PMDSU programme and PKPI PMDSU 2018.

\section{References}

[1] S. Türk, Altınsoy, G. ÇelebiEfe, M. Ipek, M. Özacar, C. Bindal, Microwave-assisted biomimetic synthesis of hydroxyapatite using different sources of calcium, Mater. Sci. Eng. C 76 (2017) 528-535, https://doi.org/10.1016/j.msec.2017.03.116.

[2] I. Bogdanoviciene, K. Tonsuaadu, V. Mikli, I. Grigoraviciute-Puroniene, A. Beganskiene, A. Kareiva, $\mathrm{pH}$ impact on the sol-gel preparation of calcium hydroxyapatite, Ca10(PO4)6(OH)2, using a novel complexing agent, DCTA, Cent. Eur. J. Chem. 8 (2010) 1323-1330, https://doi.org/10.2478/s11532-010-0113-0.

[3] N. Jamarun, Z. Azharman, S. Arief, T.P. Sari, A. Asril, S. Elfina, Effect of temperature on synthesis of hydroxyapatite from limestone, Rasayan J. Chem. 8 (2015) 133-137.

[4] A. Szcześ, L. Hołysz, E. Chibowski, Synthesis of hydroxyapatite for biomedical applications, Adv. Colloid Interface Sci. 249 (2017) 321-330, https://doi.org/10. 1016/j.cis.2017.04.007.

[5] B. Cengiz, Y. Gokce, N. Yildiz, Z. Aktas, A. Calimli, Synthesis and characterization of hydroxyapatite nanoparticles, Colloid. Surf. Physicochem. Eng. Asp 322 (2008) 29-33, https://doi.org/10.1016/j.colsurfa.2008.02.011.

[6] Y. Azis, N. Jamarun, S. Arief, H. Nur, Facile synthesis of hydroxyapatite particles from cockle shells (Anadara granosa) by hydrothermal method, Orient, J. Chem. 31 (2015) 1099-1105, https://doi.org/10.13005/ojc/310261.

[7] N. Jamarun, Miftahurrahmi, U. Septiani, Synthesis of hydroxyapatite from halaban limestone by sol-gel method, Res. J. Pharm. Biol. Chem. Sci. 7 (2016) 2956-2961.

[8] S. Jadalannagari, K. Deshmukh, S.R. Ramanan, M. Kowshik, Antimicrobial activity of hemocompatible silver doped hydroxyapatite nanoparticles synthesized by modified sol-gel technique, Appl. Nanosci. 4 (2014) 133-141, https://doi.org/10. 1007/s13204-013-0197-x.

[9] V. Stanić, D. Janaćković, S. Dimitrijević, S.B. Tanasković, M. Mitrić, M.S. Pavlović, A. Krstić, D. Jovanović, S. Raičević, Synthesis of antimicrobial monophase silverdoped hydroxyapatite nanopowders for bone tissue engineering, Appl. Surf. Sci. 257 (2011) 4510-4518, https://doi.org/10.1016/j.apsusc.2010.12.113.

[10] A. Akhavan, N. Sheikh, F. Khoylou, F. Naimian, E. Ataeivarjovi, Synthesis of antimicrobial silver/hydroxyapatite nanocomposite by gamma irradiation, Radiat. Phys. Chem. 98 (2014) 46-50, https://doi.org/10.1016/j.radphyschem.2014.01. 004.

[11] A. Bharti, S. Singh, V.K. Meena, N. Goyal, Synthesis of novel multiple shaped silver nanoparticles incorporated hydroxyapatite nanocomposite for orthopaedic body implants, Adv. Sci. Lett. 20 (2014) 1297-1302, https://doi.org/10.1166/asl.2014 5508.

[12] H. Nikaido, Multidrug resistance in bacteria, Annu. Rev. Biochem. 78 (2009) 119-146, https://doi.org/10.1146/annurev.biochem.78.082907.145923. Multidrug.

[13] M.K. Rai, S. Deshmukh, A. Ingle, A.K. Gade, Silver nanoparticles: the powerful nanoweapon against multidrug-resistant bacteria, J. Appl. Microbiol. 112 (2012) 841-852, https://doi.org/10.1111/j.1365-2672.2012.05253.x.

[14] A. Mocanu, G. Furtos, S. Rapuntean, O. Horovitz, C. Flore, C. Garbo, A. Danisteanu, G. Rapuntean, C. Prejmerean, M. Tomoaia-Cotisel, Synthesis; characterization and antimicrobial effects of composites based on multi-substituted hydroxyapatite and silver nanoparticles, Appl. Surf. Sci. 298 (2014) 225-235, https://doi.org/10.1016/ j.apsusc.2014.01.166.

[15] N.A. Charlena, Handika Nuzulia, Synthesis and characterization of composite synthesis and characterization of composite hydroxyapatite-silver nanoparticles, IOP Conf. Ser. Earth Environ. Sci. 58 (2017) 1, https://doi.org/10.1088/1755$1315 / 5$.

[16] A. Labanni, Z. Zulhadjri, D. Handayani, Y. Ohya, The effect of monoethanolamine as stabilizing agent in Uncaria gambir Roxb . mediated synthesis of silver nanoparticles and its antibacterial activity, J. Dispersion Sci. Technol. (2019) 1-8, https://doi.org/10.1080/01932691.2019.1626249.

[17] A. Labanni, Zulhadjri, D. Handayani, S. Arief, Uncaria gambir Roxb. mediated green synthesis of silver nanoparticles using diethanolamine as capping agent, IOP Conf. Ser. Mater. Sci. Eng, 2018, pp. 1-6, , https://doi.org/10.1088/1757-899X/299/1/ 012067.

[18] N. Jafari, L. Karimi, M. Mirjalili, S.J. Derakhshan, Effect of Silver Particle size on color and Antibacterial properties of silk and cotton Fabrics, Fibers Polym. 17 (2016) 888-895, https://doi.org/10.1007/s12221-016-6052-4.

[19] M. Guzman, J. Dille, S. Godet, Synthesis and antibacterial activity of silver nanoparticles against gram-positive and gram-negative bacteria, Nanomed. Nanotechnol. Biol. Med. 8 (2012) 37-45, https://doi.org/10.1016/j.nano.2011.05 007.

[20] F.A.C. Andrade, L.C.D.O. Vercik, F.J. Monteiro, E.C.D.S. Rigo, Preparation, characterization and antibacterial properties of silver nanoparticles-hydroxyapatite composites by a simple and eco-friendly method, Ceram. Int. 42 (2016) 2271-2280, https://doi.org/10.1016/j.ceramint.2015.10.021.

[21] M. Mirzaee, M. Vaezi, Y. Palizdar, Synthesis and characterization of silver doped hydroxyapatite nanocomposite coatings and evaluation of their antibacterial and corrosion resistance properties in simulated body fluid, Mater. Sci. Eng. C 69 (2016) 675-684, https://doi.org/10.1016/j.msec.2016.07.057.

[22] R.P. Singh, M. Singh, G. Verma, S. Shukla, S. Singh, S. Singh, Structural analysis of silver doped hydroxyapatite nanopowders by rietveld refinement, Trans. Indian Inst. Met. 70 (2017) 1973-1980, https://doi.org/10.1007/s12666-016-1019-0. 
[23] R. Nirmala, F.A. Sheikh, M.A. Kanjwal, J.H. Lee, S. Park, R. Navamathavan, H.Y. Kim, Synthesis and characterization of bovine femur bone hydroxyapatite containing silver nanoparticles for the biomedical applications, J. Nanoparticle Res. 13 (2011) 1917-1927, https://doi.org/10.1007/s11051-010-9944-z.

[24] S.M. Magaña, P. Quintana, D.H. Aguilar, J.A. Toledo, C. Ángeles-Chávez,

M.A. Cortés, L. León, Y. Freile-Pelegrín, T. López, R.M.T. Sánchez, Antibacterial activity of montmorillonites modified with silver, J. Mol. Catal. A Chem. 281 (2008) 192-199, https://doi.org/10.1016/j.molcata.2007.10.024.

[25] M. Yamamoto, Y. Kashiwagi, M. Nakamoto, Size-controlled synthesis of monodispersed silver nanoparticles capped by long-chain alkyl carboxylates from silver carboxylate and tertiary amine, Langmuir 22 (2006) 8581-8586.

[26] J.-T. Wu, S.L.-C. Hsu, Preparation of triethylamine stabilized silver nanoparticles for low-temperature sintering, J. Nanoparticle Res. 13 (2011) 3877-3883, https://doi. org/10.1007/s11051-011-0341-z.

[27] A. Bharti, S. Singh, V.K. Meena, N. Goyal, Structural characterization of silver-hydroxyapatite nanocomposite: a bone repair biomaterial, Mater. Today Proc. 3 (2016) 2113-2120, https://doi.org/10.1016/j.matpr.2016.04.116. 